\title{
TRANSICIÓN DE LA NATALIDAD DE SILES (Jaén) EN EL SIGLO XX: Tasa bruta, proporción secundaria por sexos, gemeralidad, ilegitimidad y estacionalidad
}

\author{
Ramón Beteta Avio \\ Doctorando del Laboratorio de Antropología \\ Depto. Medicina Legal, Toxicología y \\ Antropología Física. Facultad de Medicina. \\ Universidad de Granada \\ Correo electrónico: rabeteta@yahoo.es
}

\section{Resumen}

En el presente trabajo se analiza la transición de la tasa bruta, proporción secundaria por sexos, gemelaridad, ilegitimidad y estacionalidad de la natalidad en la población de Siles (Jaén) a lo largo del siglo XX. Se ha fotografiado digitalmente e informatizado todas las actas de bautizos registradas desde 1900 a 1999 en los libros originales de la parroquia del pueblo, y tomado datos en el Registro Civil, totalizando 10.793 nacimientos. La tasa bruta ha pasado de 51,44\%。 en 1900 a $11,51 \%$ on 1999 . Se registra para la centuria una tasa de masculinidad del 102,65. Las frecuencias de gemelos presentan un índice total de gemeralidad de 8,98\%; el 4,447\%。 en la dizigótica y el 4,54\%。 en la monozigótica. La tasa global de ilegitimidad observada es del 4,52\%. La estacionalidad registra máximos en el mes de agosto y mínimos en noviembre y diciembre. Se estima que la emigración ha sido el factor que más ha influido en la transición de la natalidad.

Palabras clave: Natalidad, Siglo XX, Siles, tasa bruta, estacionalidad, proporción secundaria por sexos.

\section{Abstract}

In this work is analyzes the transition of the rate gross, proportion secondary by sexes, twin formation, illegitimacy and seasonality of the birth in the population of Siles (Jaén) along the century XX. It has been digitally photographed and computerized all the records of baptisms recorded since 1900 to 1999 in the original books of the only parish of the village, and taken data in the Civil Registry, totaling 10.793 births. The number of births went from 162 in 1900 to 29 in 1999. Is recorded for the century: a rate gross of $27,12 \%$; a rate of masculinity of the 102,65; the frequency of twins have an index of gemeralidad of $8,98 \%, 4,44 \%$ in the dizigotica- $4,54 \%$ o in the 
monozygotic; the observed illegitimacy rate is $4.52 \%$; the seasonality registers highs in the month of August and minimums in November and December. It is estimated that emigration has been the factor that has most influenced the transition from birth.

Key words: Natality, 20th century, Siles, gross rate, secondary proportion by sex, twinhood, illegitimacy, seasonality

\section{INTRODUCCIÓN}

Entre los distintos aspectos que se pueden estudiar sobre la natalidad humana desde la perspectiva de la biodemografía figuran las fases y ritmos de transición de la tasa bruta, la proporción secundaria por sexos, la gemelaridad, la ilegitimidad y la estacionalidad de la natalidad ${ }^{1}$.

Estas facetas de la natalidad registran diferencias destacadas entre poblaciones dado que dependen del influjo y convergencia de múltiples factores y de ahí la importancia de su estudio. Así, el valor de la tasa bruta depende de diversos factores: económicos; biológicos, como la precocidad e intensidad de la nupcialidad o la estructura de la población; socioculturales, como la importancia de la religión, la influencia de la administración pública en difundir políticas que fomenten el aumento o disminución del número de hijos, la aceptación social de los anticonceptivos, nivel de instrucción, el divorcio, el aborto, las guerras, etc. (Devolver et al., 2006: 78-79; Foschiatti, 2010: 5; Hawley, 1962: 125; Losada, 1999: 51; Luna, 1984: 67; Quesada, 2002: 39 entre otros). También se asocian los procesos de avances socio-económicos, como la urbanización, alfabetización, secularización, alteración del status de la mujer, etc. con la reducción de los niveles de natalidad (Arango, 1980: 186-187) y se debe tener en cuenta la migración femenina como un factor en la transición de la natalidad de las poblaciones rurales (Del Rey et al., 2010: 115).

El segundo aspecto, la proporción secundaria por sexos, o proporción de sexos al nacimiento, depende de la proporción primaria y de la influencia de complejos factores, entre los que destacan los fisiológicos, biodemográficos y socioeconómicos (Fellman y Ericsson, 2009: 105-107; Jacobsen et al., 1999: 3122-3124; Quesada, 2002: 66-72; Sánchez Compadre, 1989: 58). Asimismo, autores como Bertranpetit y Sala (1987: 177) y Luna (1984: 69) se preguntan por la existencia de factores

\footnotetext{
${ }^{1}$ El contenido de este articulo forma parte de una tesis doctoral en la que se está trabajando sobre la evolución biodemográfica en el siglo XX de la población de Siles (Jaén).
}

Publicado en formato digital: Ramón Beteta Avio. TRANSICIÓN DE LA NATALIDAD DE SILES (Jaén) EN EL SIGLO XX: Tasa bruta, proporción secundaria por sexos, gemeralidad, ilegitimidad y estacionalidad. Revista Geográfica Digital. IGUNNE. Facultad de Humanidades. UNNE. Año 14. № 27. Enero - Junio 2017. ISSN 1668-5180 Resistencia, Chaco.

En: http://hum.unne.edu.ar/revistas/geoweb/default.htm 
genéticos que puedan explicar los diferentes resultados registrados en las poblaciones.

Los partos gemelares constituyen un aspecto biológico de gran interés. Las causas por las que se producen son bastante heterogéneas y poco conocidas. No obstante, existen estudios que citan autores como Fuster et al. (2006: 19 y 2009:2), Luna (1984: 83-86), Quesada (2002: 86-87), Sánchez Compadre (1989: 61), Rodríguez (1984: 105), Valls (1972: 238), entre otros, que demuestran que las frecuencias de los partos gemelares dizigóticos (dos óvulos) están influenciadas por diversos factores: genéticos, la edad de la madre, la paridad, la nutrición, la reproducción asistida y el índice de masa corporal ${ }^{2}$. La gemeralidad monozigótica (un óvulo) es debida a la acción del azar, e influyen también factores ambientales (ningún autor menciona factores genéticos para este tipo de gemeralidad).

La ilegitimidad constituye una faceta de la natalidad muy estudiada por la información que aporta sobre los cambios socioculturales y económicos de las poblaciones humanas. Las diferencias entre las frecuencias de las distintas poblaciones radican en factores como la edad de la madre al matrimonio, la zona de residencia, las diferencias culturales y educacionales, las diferencias económicas y sociales, y el grado de religiosidad (Alfonso-Sánchez y Peña (2003: 60); Camarota (2007: 2-3); García-Moro, 1986: 75; Rodríguez, 1984:101; Sánchez Compadre (1989: 62)). Díez (1984: 97-99) menciona que a finales del siglo XX el aumento de la ilegitimidad es producto de dos variables fundamentales: un cambio de las actitudes sociales ante las relaciones sexuales prematrimoniales y una gradual tendencia a posponer el matrimonio. Este mismo autor menciona a Goode, para añadir un nuevo factor que sería el comportamiento anómico de la mujer para "cazar" marido, añade que este fenómeno es posiblemente característico de sociedades tradicionales y de los estratos más bajos de la población.

La última faceta, la estacionalidad de la natalidad, es consecuencia de la estacionalidad de las concepciones. Por tanto, para inferir sobre las causas de la variación estacional de los nacimientos es imprescindible conocer los distintos factores

\footnotetext{
${ }^{2}$ María de la Calle, jefa de la Unidad de Gestaciones Múltiples del Hospital de la Paz (de referencia para estos tipos de partos), en una entrevista que concedió a Victoria Torres Benayas publicada por el diario El PAIS del 16 de mayo de 2016, menciona el índice de masa corporal como un factor que favorece los embarazos gemelares (las mujeres más gordas y más altas tienen mayor propensión a concebir embarazos gemelares).
}

Publicado en formato digital: Ramón Beteta Avio. TRANSICIÓN DE LA NATALIDAD DE SILES (Jaén) EN EL SIGLO XX: Tasa bruta, proporción secundaria por sexos, gemeralidad, ilegitimidad y estacionalidad. Revista Geográfica Digital. IGUNNE. Facultad de Humanidades. UNNE. Año 14. № 27. Enero - Junio 2017. ISSN 1668-5180 Resistencia, Chaco.

En: http://hum.unne.edu.ar/revistas/geoweb/default.htm 
que operan en la estacionalidad de las concepciones. Entre éstos figuran biológicos, climáticos, sociales y culturales (Hernández et al. 2013: 37; Luna, 1984: 74; Martínez Carrión, (1984: 101-102); Quesada, 2000: 130 y 2002: 44; Sánchez Compadre, 1989: $57)$.

El siglo $\mathrm{XX}$ ha protagonizado las mayores transformaciones sociales, económicas y culturales de la historia, y ellas han influido significativamente en la evolución de la natalidad. En el caso concreto de España y, sobre todo, en las áreas rurales estas transformaciones han marcado de forma fundamental la evolución de la natalidad. Por ello es de notable interés el análisis de la evolución de las distintas facetas de la natalidad en poblaciones rurales de distintas regiones. Con esta premisa, se ha planteado como objetivo principal de este trabajo el análisis de la evolución de la natalidad en una población rural aislada de Andalucía a lo largo de todo el siglo XX. El espacio seleccionado ha sido Siles, una localidad de la comarca natural de la Sierra de Segura (Jaén), que ha presentado un grado elevado de aislamiento, explicado por factores geográficos y socio-culturales que han condicionado la vida cotidiana de sus pobladores.

\section{MATERIAL Y METODOS}

Siles (Latitud: $38^{0} 23^{\prime}$ Norte / Longitud: $2^{0} 35^{\prime}$ Oeste) es una villa de la provincia de Jaén, partido judicial de Villacarrillo. Está ubicada a 828 metros de altitud, al Noreste de la Sierra de Segura y de la provincia. Posee una extensión de 175,87 $\mathrm{Km}^{2}$, de los que 165,66 están incluidos dentro del mayor espacio protegido de España y el segundo de Europa, el Parque Natural de Cazorla, Segura y las Villas. La población censal media de Siles en el siglo XX ha sido de 3.748 habitantes. Su economía durante este siglo ha estado basada en el cultivo del olivo y la plantación y corta de pinos. Ambas han experimentado una gran transformación a lo largo de la centuria, así, el cultivo del olivo ha aumentado su productividad pasando de los $70 \mathrm{~kg} /$ pareja/día en la recogida preindustrial a $400 \mathrm{~kg} /$ persona/día con las vibradoras de las décadas finales (Infante, 2011: 111); y la declaración de Parque Natural y la mecanización han influido en el número de jornales que daba en el pueblo la plantación y corta de pinos.

El estudio sobre la natalidad sileña tiene como principal fuente de datos los libros originales de bautismo de la única parroquia del pueblo. Se han fotografiado 
digitalmente todas las actas de bautizos registradas desde 1900 a 1999. Posteriormente, se han informatizado con el programa Access de Microsoft. En total figuran 10.793 fichas individuales con todos los datos de interés demográfico ${ }^{3}$, que corresponden a los nacimientos de 5.342 mujeres y 5.445 varones, más 6 sujetos sin sexo definido. Para la década de 1930 - 39 se han anexionado los datos del Registro Civil. Como señala Bertranpetit (1978: 411) el registro religioso de nacimientos es a veces mejor que el Civil, dado que hasta el año 1991 (ley 18/1990, del 17 de diciembre sobre la reforma del Código Civil, en la que se índica que los nacimientos deben ser registrados en el lugar de residencia de la madre) se registraban los bebés en el lugar en donde se producía el parto y esto provocó que muchos nacimientos de zonas rurales se registrasen en las ciudades en donde estaban ubicados los hospitales. Se ha comprobado que el paso de un Estado confesional a uno laico con la Constitución de 1978 apenas produjo modificaciones en las prácticas religiosas de la población sileña en las dos últimas décadas del siglo. Para ello se han cotejado los registros civil y religioso en los años 1982, 1988 y 1994 en donde las diferencias encontradas entre ambos registros no resultaron significativas.

Se ha optado por el cómputo de la población de hecho, ofrecida por los censos, como el más ajustado a la población residente de Siles a lo largo del siglo XX. De los 11 censos que se utilizan, los 8 correspondientes a los años acabados en cero están referidos al 31 de diciembre. Esta fecha coincide con los meses del año en los que se realiza la recolección de la aceituna por lo que los jornaleros agrícolas sileños se encuentran en el pueblo. Para el cálculo del número de habitantes al $1^{\circ}$ de julio de los años intercensales se ha utilizado la formula de crecimiento geométrico o compuesto (tasa de crecimiento anual acumulativo) expuesta y desarrollada por Livi-Bacci (1993: 37).

Las tasas brutas de natalidad, nupcialidad y mortalidad, así como, los índices de mortalidad infantil y de párvulos ${ }^{4}$, y las edades medias al matrimonio han sido calculados siguiendo lo expuesto por Rodríguez (1984: 24 y 59). Las tasas brutas de natalidad por quinquenios son los promedios de las tasas de los años que componen el quinquenio (Luna, 1984: 63). En el estudio de la proporción secundaria por sexos se emplea la tasa de masculinidad o "proporción de varones al nacimiento" (Rodríguez

\footnotetext{
${ }^{3}$ Los campos utilizados para este trabajo son: gemelaridad, ilegitimidad, sexo, mes y año del nacimiento.

${ }^{4}$ Párvulos: 1 - 4 años.
}

Publicado en formato digital: Ramón Beteta Avio. TRANSICIÓN DE LA NATALIDAD DE SILES (Jaén) EN EL SIGLO XX: Tasa bruta, proporción secundaria por sexos, gemeralidad, ilegitimidad y estacionalidad. Revista Geográfica Digital. IGUNNE. Facultad de Humanidades. UNNE. Año 14. № 27. Enero - Junio 2017. ISSN 1668-5180 Resistencia, Chaco.

En: http://hum.unne.edu.ar/revistas/geoweb/default.htm 
1984: 99). En el análisis de la gemeralidad se utiliza método diferencial de Weimberg ${ }^{5}$ (Valls, 1972: 236) ${ }^{6}$. Las tasas de ilegitimidad se calculan en base al número de nacimientos de hijos habidos fuera del matrimonio por cada 100 nacimientos del periodo considerado (Sánchez Compadre, 1989: 61). Para analizar la estacionalidad de la natalidad sileña se ha divido el siglo en veintenas y se emplea el coeficiente de estacionalidad de Henry (1976: 57). Al carecer de datos directos sobre los movimientos migratorios se han calculado los saldos migratorios sileños de forma indirecta, según indica Henry (1983: 248).

Los datos utilizados sobre las tasas brutas de natalidad de España proceden de Nadal (1984: 140-141) hasta el año 1980 y los de los años posteriores del INEbase; los de Andalucía y la provincia de Jaén se encuentran en la Web del IECA; y los de la ciudad de Jaén provienen del INE (1956: 92).

Se ha utilizado el test de "chi cuadrado" mediante el programa informático SSPS 15.0 para comprobar el ajuste de los datos registrados a las distribuciones teóricas.

\section{RESULTADOS Y DISCUSIÓN}

\section{I.- EVOLUCIÓN GENERAL DE LA NATALIDAD}

En la figura 1 se representa la evolución anual del número de nacimientos. Siles ha pasado de 162 nacimientos registrados en el año 1900, a 114 en 1950, 52 en 1970 y a 29 en el año 1999. En conjunto se ha registrado un descenso en el número de nacimientos del $82,1 \%$ entre el primer y el último año del siglo. Los años que registran menor número de nacimientos son 1997 con 20 y 1991 con 23. Por el contrario, los años que registran mayor número son 1940 con 201 y 1933 con 183.

En la primera mitad del siglo la natalidad sileña oscila en torno a los 150 nacimientos anuales. La oscilación más significativa se registra entre los años de 1939 y 1940, al pasar de 117 nacimientos en el primer año a 201 en el segundo, con un repunte del $71,8 \%$. El descenso de la natalidad en los años de la Guerra Civil, el

\footnotetext{
${ }^{5}$ Fellman y Eriksson (2006) concluyen que los resultados obtenidos empleando el método Weimberg son bastante fiables.

${ }^{6}$ Valls (1972: 237) emplea una formula que tiene en cuenta la mayor probabilidad de nacer varón y prácticamente obtiene los mismos resultados. Aplicada por autores como Sánchez Compadre (1989: 60), Luna (1984: 84) y Alfonso-Sánchez y Peña (2003: 58) observamos que registran mínimas diferencias a los resultados sin corregir, por lo que decidimos no emplearla.
}

Publicado en formato digital: Ramón Beteta Avio. TRANSICIÓN DE LA NATALIDAD DE SILES (Jaén) EN EL SIGLO XX: Tasa bruta, proporción secundaria por sexos, gemeralidad, ilegitimidad y estacionalidad. Revista Geográfica Digital. IGUNNE. Facultad de Humanidades. UNNE. Año 14. № 27. Enero - Junio 2017. ISSN 1668-5180 Resistencia, Chaco.

En: http://hum.unne.edu.ar/revistas/geoweb/default.htm 
brusco repunte del año 1940, la posterior caída en 1941 y la recuperación en 1942, coinciden con las registradas en las poblaciones de España, Andalucía, provincia y ciudad de Jaén.

Desde el año 1947 al 1952 se registra un significativo descenso en el número de nacimientos sileños. En este periodo se pasó de 160 nacimientos al año a 95, lo que supone una bajada del 40,6\%. Los habitantes disminuyeron el 3,1\%, de 4.946 a 4.793. El descenso del número de nacimientos en este quinquenio se debe, en cierta medida, al descenso del 27,5\% en las frecuencias de nupcialidad (si contabilizamos el año 1946 es del 60\%) y a la emigración iniciada en los años de la posguerra (en este lustro se registra un saldo migratorio negativo de 469 personas). Otro descenso importante en el número de nacimientos sileños se registró en los años 1969 y 1970. Es el descenso más brusco del siglo sumando dos años consecutivos. De 84 nacimientos en el año 1968 se pasó a 52 en 1970, un descenso del 38,1\%, mientras que el número de habitantes solo descendió un 3,2\% entre estos años, y la tasa bruta de nupcialidad repunto un $24,2 \%$. El hecho de que coincida una importante bajada de los nacimientos con un importante repunte en los matrimonios indicaría una menor fecundidad de las parejas, que reducen el número de hijos. En la última década del siglo la natalidad sileña alcanza las cotas más bajas de nacimientos, descendiendo de los 25 en los años 1991, 1996 y 1997.

Para evitar las continuas oscilaciones y poder observar las tendencias ocultas, además de facilitar su interpretación, se han promediado las tasas brutas para periodos de cinco años, tal como hacen otros autores (Bertranpetit, 1978: 411; Luna, 1984:63).

En la evolución por quinquenios de la tasa de natalidad sileña (figura 2) se han diferenciado tres periodos, de 1900/04 a 1920/24, de 1925/29 a 1965/69 y de 1970/74 a 1995/99.

El primer periodo se caracteriza por tasas brutas de natalidad altas ${ }^{7}$. Registra una tasa media de $42,7 \%$ o, con la mayoría de los años por encima del $40 \%$. Estas altas tasas de natalidad se deben a la convergencia e influencia de numerosos factores, entre los que destacan la alta y temprana nupcialidad (tasa bruta media de nupcialidad para el periodo 7,9\%; edad media al matrimonio: 27 años en el varón y 23 en la mujer), una estructura poblacional con amplios grupos de individuos en edades fértiles

\footnotetext{
${ }^{7}$ Los umbrales de intensidad de la tasa bruta de natalidad proceden de Foschiatti (2010: 3).
} 
(aún no se ha registrado ningún movimiento migratorio significativo ${ }^{8}$ ), el alto valor de los hijos en la sociedad agrícola y el sentimiento pronatalista de la población ante una elevada mortalidad infantil y de párvulos (índice de mortalidad infantil medio para el periodo: 168\%; de párvulos: 166,5\%o). Este primer periodo coincide con tasas brutas de mortalidad también altas $(28,5 \%$ ). Las tasas brutas altas son una característica común de las zonas subdesarrolladas e indicativo de que durante este periodo aún regía en la población de Siles el régimen demográfico antiguo. Desde 1900-04 a 19151919 la tasa bruta de natalidad sileña desciende de forma continua, registrándose una caída del $23,6 \%$ en el cuarto quinquenio con relación al primero. En el quinto quinquenio la tasa repunta un 7,3\% con relación al anterior. Las trayectorias de las tasas brutas de natalidad española, andaluza y giennense realizan similar oscilación, bajada paulatina a lo largo de los primeros cuatro quinquenios y repunte en el quinto, el posterior a la "gripe española". En España entre el primer y cuarto quinquenio la tasa descendió un 16,6\%, y ascendió en el quinto el 3\%. En Andalucía ${ }^{9}$, para los mismos quinquenios, registró un descenso del 14,4\% y después repuntó un $3,7 \%$. En la provincia de Jaén cayó $14,1 \%$ y repuntó un 1,1\% y en la ciudad de Jaén descendió un $15,3 \%$ y repuntó un $8 \%$. Se observa que en los primeros veinte años del siglo las tasas brutas de natalidad descienden más en Siles que en las otras poblaciones, lo que puede deberse al pequeño tamaño de la población.

Durante este periodo la bajada de la tasa bruta de natalidad en Siles fue provocada por el incremento en el número de habitantes. Entre los años 1900 y 1924 , primero y último de este periodo, la tasa bajó en Siles un $27,7 \%$; el número de habitantes se incrementó un $34 \%$ y el número de nacimientos repuntó un 1,8\%. El que no subiera el número de nacimientos de forma similar al número de habitantes se debe entre otras causas al escaso repunte de los matrimonios (el 1,7\% en este periodo) y el aumento del porcentaje de población soltera (pasó del 52,8\% en el censo de 1900 al $58,5 \%$ en el de 1920). Asimismo, como señala Nadal (1984: 217), existe un punto de inflexión en el año 1914 cuando la natalidad española inicia su entrada en el circulo de la natalidad europea (descenso de la mortalidad, dificultades de emigrar a América, proceso de urbanización en el país) limitándose voluntariamente el número de hijos en

\footnotetext{
${ }^{8}$ Infante (2011: 92- 93) indica que en estas primeras décadas del siglo XX se produce una fase expansiva del olivar que requirió importantes cantidades de mano de obra tanto masculina como femenina, comenta que en el año 1900 la superficie de olivar en Andalucía era de 743.000 hectáreas y, antes de la guerra alcazaba el millón.

${ }^{9}$ Gil Alonso (1997: 40) encuentra diferencias entre la Andalucía oriental, de nupcialidad y fecundidad más intensas, y la occidental.
}

Publicado en formato digital: Ramón Beteta Avio. TRANSICIÓN DE LA NATALIDAD DE SILES (Jaén) EN EL SIGLO XX: Tasa bruta, proporción secundaria por sexos, gemeralidad, ilegitimidad y estacionalidad. Revista Geográfica Digital. IGUNNE. Facultad de Humanidades. UNNE. Año 14. № 27. Enero - Junio 2017. ISSN 1668-5180 Resistencia, Chaco.

En: http://hum.unne.edu.ar/revistas/geoweb/default.htm 
la familia. También Sanz y González (2001: 65) registran en la población de Aranjuez el inicio de la consolidación del control de la fecundidad en las primeras décadas del siglo. Ellos comentan que la paridad media total inicia su descenso en el quinquenio 1911/15 y el de la edad media del último hijo en el de 1921/25.

El segundo periodo del siglo (1925/29-1965/-69) se caracteriza por tasas brutas de natalidad intermedia. A lo largo de este periodo la tasa de natalidad sileña registró un descenso del 42,4\%, y una tasa media del 27,74\%. El número de habitantes aumentó hasta el año 1941, a partir del cual fue descendiendo para marcar el mínimo a finales de la centuria. La cifra de habitantes ascendió desde el quinquenio de 1925/29 al de 1940/44 un 16\%, para bajar a continuación un 23.5\% hasta el quinquenio de 1965/69. El número de nacimientos pasó de 775 en el quinquenio de 1925/29 a 397 en el de 1965/69; un descenso del 48,8\%. Entre el primer y último quinquenios de este periodo la tasa bruta de natalidad española bajó un $28,7 \%$, la de Andalucía un $28 \%$ y la provincia de Jaén un 39,7\%. Este descenso se registra también en otras poblaciones rurales españolas (Luna, 1984: 65; Rodríguez, 1984: 93; Sánchez-Compadre, 1989: 55). En los nueve quinquenios que componen este periodo solo se registra un repunte del índice en el lustro de 1960/64. El "baby boom" sileño supuso un incremento en la tasa del 5,7\% con relación al quinquenio anterior. En este mismo quinquenio la tasa de natalidad española repuntó un 0,8\%, la de Andalucía un $1,1 \%$ y la de la provincia de Jaén un $3 \%$.

Dentro de este periodo se diferencian claramente dos subperiodos: el primero desde 1925/29 a 1945/49, con una tasa bruta de natalidad medía del 32,77\%。 (intermedia alta), y el segundo subperiodo, desde 1950/54 a 1965/69, con una tasa media del 21,45\%。 (intermedia baja). En el primer subperiodo destaca la década de la posguerra de 1940/49 en la que el índice de natalidad sileño se mantiene relativamente alto, en torno al $30 \%$, a pesar de las tremendas penurias por las que atraviesa la población y el inicio de los movimientos migratorios. En el segundo subperiodo se produce el inicio de la modernización socio-económica del pueblo (canalización de las aguas y electrificación de las viviendas, modernización de la agricultura, mayor poder adquisitivo de los ciudadanos, mejora de las comunicaciones, mayor nivel de instrucción y cultural, primeros electrodomésticos, etc.). Al igual que en otras zonas rurales, como La Alpujarra, Ancares o Babia (Luna, 1984; Rodríguez, 1984; Sánchez-Compadre, 1989), el factor que más influyó en el descenso registrado por la tasa de natalidad sileña en este periodo fue el gran movimiento migratorio de 
parte de su población a zonas urbanas e industrializadas, que ocasionó la pérdida de población que integran los grupos de edades fértiles y, en consecuencia, el descenso del número de nacimientos. En los 30 años de las décadas 40, 50 y 60 la población de Siles registró un saldo migratorio negativo de 3.121 personas, de las que 1.723 fueron mujeres, el 55,2\%. Otro factor que influyó en el descenso de la natalidad en este periodo fue la bajada de la mortalidad infantil y de párvulos (el índice de mortalidad infantil descendió del $129,03 \%$ en el quinquenio de 1925/29 al 27,7\% en el de 1965/69; y el de párvulos pasó del 132,9\%。 en el quinquenio de 1925/29 al 10\%。 en el de 1960/64). Como menciona Devolver et al. (2006: 79), esto provocó que cada vez fuese menos necesario para las familias tener nacimientos de reemplazo, es decir segundos, terceros o nacimientos de orden superior que pudieran suplir la muerte de un hermano/a mayor. También debió influir en el descenso de la natalidad sileña la difusión de la limitación de la fecundidad; Sanz y González (2001: 68) comentan como en la población de Aranjuez llegó al 65\% de las mujeres en el quinquenio de 1966/70 Asimismo, los avances socio-económicos mencionados para el segundo subperiodo influyeron en el descenso de la mortalidad y la natalidad sileñas.

El tercer periodo (1970/1974-1995/1999) muestra tasas brutas de natalidad bajas. En él se registra un descenso de la tasa de natalidad del $27 \%$, y una tasa media del 13,21\%. El número de habitantes descendió de 3.688 en el año 1970 a 2.506 en 1999, por lo que cayó el 32,1\%. El número de nacimientos descendió de 52 en el año 1970 a 29 en 1999, un 44,3\%. En este periodo la tasa bruta de natalidad española descendió un $52,1 \%$, la andaluza un $48,7 \%$ y la tasa provincial un $38,5 \%$. Los promedios fueron de un $13,51 \%$ on la española, un $15,78 \%$ en la andaluza y de un 14,52\% en la provincia de Jaén. La población de Siles en la década de 1970/79 registra un significativo saldo migratorio negativo de 788 individuos, 318 mujeres y 470 varones. Esto propició que durante estos años la tasa de natalidad continuase descendiendo con fuerza. El repunte del índice de natalidad sileño en el quinquenio de 1980/84, y su mantenimiento en el posterior, se estima que se debe a una situación socio-económica favorable. Egea (1999: 224) registra un aumento similar de la natalidad en varias poblaciones de la provincia de Jaén en la década de 1981/1991, que relaciona con su desarrollo económico. Asimismo, el ligero aumento de las tasas sileñas en estos años se puede considerar normal dentro de las oscilaciones que registra el patrón general de una natalidad a la baja. En la última década del siglo, 1990/99, vuelve a bajar la ya exigua natalidad sileña (la tasa bruta desciende el 25,2\%

Publicado en formato digital: Ramón Beteta Avio. TRANSICIÓN DE LA NATALIDAD DE SILES (Jaén) EN EL SIGLO XX: Tasa bruta, proporción secundaria por sexos, gemeralidad, ilegitimidad y estacionalidad. Revista Geográfica Digital. IGUNNE. Facultad de Humanidades. UNNE. Año 14. № 27. Enero - Junio 2017. ISSN 1668-5180 Resistencia, Chaco.

En: http://hum.unne.edu.ar/revistas/geoweb/default.htm 
y el número de nacimientos el 29,9\%, con relación a su anterior década) aunque se registra el importante descenso del $62,6 \%$ en el flujo migratorio negativo. Este descenso de la natalidad está relacionado con las altas tasas de celibato definitivo ${ }^{10}$ registradas en la década, el $15,03 \%$ en las mujeres y el $12,09 \%$ en los varones ${ }^{11}$. También ha influido el aumento de la edad media al matrimonio (repuntó sobre la década anterior: el 7,8\% en mujeres y el 6,1\% en varones ${ }^{12}$ ). Losada (1999: 57 ) observa como factores explicativos de las diferencias del nivel de fecundidad de las mujeres de La Habana y Oriente, las distintas edades al matrimonio y la desigual proporción que alcanza el celibato permanente.

En la tabla 1 se exponen los resultados de las tasas brutas de otras poblaciones. Entre ellas, los índices registrados por Luna (1984: 65) para la Alpujarra son los más parecidos a los sileños tanto en su evolución a lo largo del siglo como en los valores de las tasas. No obstante, en los primeros 20 años las tasas son claramente mayores en la Alpujarra, es por esto que la caída de la natalidad sileña entre los quinquenios 1900/04 y 1975/79 es algo más suave que la alpujarreña. Esta similitud puede venir dada por la semejanza de poblamiento rural en áreas montañosas relativamente aisladas. Se ha sugerido que las zonas rurales registran índices de natalidad más elevados debido a que el acceso al matrimonio se realiza en edades más tempranas y a una menor incorporación de la mujer al trabajo extradomestico. No obstante, como se puede observar en la década de los años 70 , la emigración hace que las tasas de natalidad bajen antes en las zonas rurales que en las urbanas.

En la figura 3 sobre las trayectorias de las tasas de natalidad promediada por quinquenios se observa que las caídas de las tasas nacionales, andaluzas y provinciales son muy parecidas entre si y prácticamente discurren en paralelo, sobre todo la nacional y la andaluza (en la década de los 70 las tasas provinciales descienden más que las de España y Andalucía; en los quinquenios finales del siglo volverá a dar tasas más altas). Al comparar las caídas de las tasas brutas de natalidad

\footnotetext{
${ }^{10}$ La tasa de celibato definitivo utilizada es la proporción de individuos solteros/as que mueren mayores de 50 años sobre el número total de individuos que fallecen con más de esa edad (Sánchez Compadre 1989: 79).

${ }_{11}^{11}$ Las tasas repuntaron sobre la década anterior el 3,4\% en mujeres y el $81,2 \%$ en varones.

12 Las edades medias al matrimonio fueron en la década 1980/89 de 22,9 años en la mujer y 26 en el varón; en la década de 1990/99 fueron 24,7 años en la mujer y 27,6 en el varón
}

Publicado en formato digital: Ramón Beteta Avio. TRANSICIÓN DE LA NATALIDAD DE SILES (Jaén) EN EL SIGLO XX: Tasa bruta, proporción secundaria por sexos, gemeralidad, ilegitimidad y estacionalidad. Revista Geográfica Digital. IGUNNE. Facultad de Humanidades. UNNE. Año 14. № 27. Enero - Junio 2017. ISSN 1668-5180 Resistencia, Chaco.

En: http://hum.unne.edu.ar/revistas/geoweb/default.htm 
entre el primer quinquenio y el último ${ }^{13}$, se observa que en España caen un $73,4 \%$, en Andalucía, un 70,8\%, en la Provincia de Jaén un 72,5\% y, finalmente, Siles registra la mayor caída, un 79,18\%. Una característica común de estas poblaciones es la de registrar las tasas más elevadas a principios de siglo. Después, se van reduciendo los valores y las diferencias entre ellas a medida que la transición de la natalidad va concluyendo, para situarse en torno al $10 \%$ a finales de siglo y presentar todas ellas un régimen demográfico moderno.

Se observa que en la década del "baby-boom" español, 1955/1964, se registra un aumento de natalidad en todas las poblaciones (Siles solo sube en el segundo quinquenio de la década), que coincide con la desaparición del bloqueo internacional y con reformas en la política económica que favorecen el crecimiento y proporcionan relativa estabilidad. Comenta Arroyo (1999: 26) que se da una coyuntura más favorable que posibilita "por un lado que las cohortes más antiguas completen en esta época sus planes familiares y por otro que las cohortes más jóvenes, dada las mayores oportunidades para obtener empleo y conseguir vivienda, comiencen antes su ciclo familiar con un matrimonio y una maternidad más precoces". Devolver et al. (2006: 78) señalan que estos aumentos de la natalidad podrían corresponder a mejoras en el nivel sanitario y nutricional de las mujeres que permitió la reducción de la mortalidad infantil y una reducción de los niveles de esterilidad asociados a las enfermedades de transmisión sexual (generalización de los antibióticos en los años 40).

El descenso de la natalidad en España en el siglo XX es un tema que continúa generando controversia debido a la cantidad de factores que han influido. No obstante, hay tres causas en las que todos los autores coinciden: la incorporación de la mujer al mundo del trabajo extradoméstico ${ }^{14}$, el desarrollo de métodos anticonceptivos y el

\footnotetext{
${ }^{13}$ Proporción del valor de la tasa del último quinquenio en relación al total de la tasa del primero. Por ejemplo, la tasa de natalidad española en el primer quinquenio del siglo, de 1900-1904, fue 34,86\% y en el último, de 1995-1999, el 9,28\%, resultando esta última tasa un $26,6 \%$ de la primera.

${ }^{14}$ Aguinaga (1989: 15) presenta unos resultados, extraídos de las Encuestas de Fecundidad realizadas por el INE en los años 1977 y 1985, en donde se observa que las proporciones de mujeres españolas casadas con trabajo pasan de ser el 25,7\% en el año 1977 al 32.5\% en 1985. Las que buscan empleo aumentan del $1,6 \%$ al 3,1\%, y las que dedican a las labores del hogar descienden del $70,8 \%$ al $63,7 \%$.
}

Publicado en formato digital: Ramón Beteta Avio. TRANSICIÓN DE LA NATALIDAD DE SILES (Jaén) EN EL SIGLO XX: Tasa bruta, proporción secundaria por sexos, gemeralidad, ilegitimidad y estacionalidad. Revista Geográfica Digital. IGUNNE. Facultad de Humanidades. UNNE. Año 14. № 27. Enero - Junio 2017. ISSN 1668-5180 Resistencia, Chaco.

En: http://hum.unne.edu.ar/revistas/geoweb/default.htm 
cambio de actitud hacia ellos ${ }^{15}$ y las crisis económicas, que traen desempleo y con ello el retraso del matrimonio y los nacimientos a épocas mejores.

\section{II.- PROPORCIÓN SECUNDARIA POR SEXOS}

En la evolución del número de nacimientos sileños por décadas y sexos (tabla 2) se observa que en la primera mitad del siglo destaca la década de 1910/19 por registrar la mayor diferencia de nacimientos entre sexos (54\% de varones). Esta mayoría de nacimientos masculinos se ve compensada en las tres décadas siguientes en las que nacen más mujeres que varones. Concretamente, en la de 1920/29 el 51\% de los nacimientos fueron mujeres, en la de 1930/39 éstas constituyeron el 50,4\%, y en la década de 1940-49 suponen 51\%. La segunda mitad del siglo registra un descenso continuo y pronunciado en el número de nacimientos, similar en ambos sexos; las diferencias por sexos a destacar se registran en la década de 1950/59 en la que el $52,7 \%$ de los nacimientos son varones y, en la siguiente de 1960/69 en la que se compensa la década anterior y nace el 51,4\% de mujeres. La dudosa correlación entre la proporción secundaria por sexos y los periodos de conflicto armado, se observa que en la población de Siles en el quinquenio de la Guerra Civil se registran menos nacimientos con mayoría de varones, el 52,5\%, y en el de la posguerra, aumenta la frecuencia de nacimientos y se registra mayoría de partos de mujeres, el $52,4 \%$. Clarkin (2011: 3) menciona que durante la guerra de Irak-Iran (1980/88) y la de Eslovenia (26 de junio al 7 de julio de 1991) bajaron las tasas de masculinidad y que se relaciona con la tensión psicológica en el hombre y sus efectos negativos en el esperma; contrariamente en la posguerra las tasas aumentan por adaptabilidad y efectos medioambientales. Brian y Jaisson (2005: 28 y 32) registran dos picos en las tasas de masculinidad de la población francesa en los años de guerra (1916/20 y 1941/45) que van parejos a una disminución de la natalidad, y una desaceleración de la masculinidad y aumento de la natalidad en la posguerra. Rodríguez (1984: 100) en los Ancares registra en la década de 1930/39 un repunte de la tasa de masculinidad y un descenso del número de nacimientos; en la década de 1940/49 vuelve a registrar un repunte de la tasa y un descenso de los nacimientos. Estos resultados

\footnotetext{
${ }^{15}$ Aguinaga (1989: 18) expone resultados con relación a las proporciones de mujeres españolas que no han utilizado métodos anticonceptivos, estas descienden del $40.2 \%$ en el año 1977 al $16,5 \%$ en 1987 , y las que han utilizado un método eficaz repunta del $25,1 \%$ al $65,5 \%$.
} 
contradictorios indicarían una relación poco significativa entre los periodos de guerra y la proporción sexual secundaria.

\section{II.- 1.- Tasa de masculinidad}

Es popularmente conocido el hecho, ya constatado, de que en la especie humana nacen más varones que mujeres; aproximadamente por cada 100 mujeres nacen 106 varones. La tasa media por nacimientos registrada para el siglo XX en Siles es de 102,65 varones por cada 100 mujeres. Como se ha mencionado, la mayor diferencia entre sexos se da en la década de 1910/19 $(117,83)$ y en la de 1950/59 $(111,65)$, en las que la tasa registra una sobrenatalidad de varones. La década de 1960/69, con 94,33 nacimientos de varones por cada 100 mujeres, es la que registra la mayor sobrenatalidad de mujeres.

En la tabla 3 se observa que las poblaciones con elevado número de nacimientos como Jaén, Andalucía y España registran unas proporciones de masculinidad sin oscilaciones bruscas ni décadas en las que las proporciones de mujeres sean mayores a los varones. Las tasas de masculinidad en estas poblaciones descienden de forma paulatina y similar hasta la década de 1950/59. A partir de la siguiente década, 1960/69, comienzan un ligero ascenso hasta la de 1980/89. En la última década de siglo prácticamente se mantienen igual a la anterior. Los resultados de las proporciones de sexo al nacer en las poblaciones rurales (tabla 3 ) registran bruscas oscilaciones y presentan décadas con mayor proporción de nacimientos de mujeres. Estos resultados tan diferentes de las poblaciones rurales estimamos que se deben a que están calculados sobre un conjunto de datos pequeño. No obstante, en estas oscilaciones se puede observar el valor adaptativo de la "sex ratio". Así, se aprecia como una oscilación ocasionada por la desviación de la tasa de masculinidad hacia uno de los sexos es compensada con otra con mayor número de nacimientos del sexo contrario, de forma que se mantiene el nivel de fecundidad de la población.

Por falta de datos no se entra a valorar la influencia en la proporción secundaría por sexos sileña de factores como las edades materna o paterna, la paridad, orden de nacimiento, el periodo fértil de la mujer, etc. Asimismo, debido a que utilizamos un número de nacimientos relativamente pequeño los resultados tienen un amplio rango de confianza para una tasa de masculinidad de 106 y un nivel de seguridad del 95\%. Luna (1984:70-71) indica que "con un nivel de seguridad del 95\%, necesitaríamos un millón de nacimientos para que una proporción de sexos de 106,

Publicado en formato digital: Ramón Beteta Avio. TRANSICIÓN DE LA NATALIDAD DE SILES (Jaén) EN EL SIGLO XX: Tasa bruta, proporción secundaria por sexos, gemeralidad, ilegitimidad y estacionalidad. Revista Geográfica Digital. IGUNNE. Facultad de Humanidades. UNNE. Año 14. № 27. Enero - Junio 2017. ISSN 1668-5180 Resistencia, Chaco.

En: http://hum.unne.edu.ar/revistas/geoweb/default.htm 
estuviese comprendida entre unos límites de confianza de 105.5 y 106.5”, y expone la escala de los límites de confianza que les corresponde a cada número de nacimientos. La población sileña, con 10.793 nacimientos en la centuria y una tasa de masculinidad de 102,65 varones por cada 100 mujeres, por décimas no se sitúa dentro del intervalo de confianza en una proporción de sexos de 106, con un nivel de seguridad del $95 \%$. Sin embargo, sí están dentro de este intervalo las tasas por décadas. Las tasas más próximas a los extremos son, por la parte alta, la década 1910/19 con 1503 nacimientos y una tasa de masculinidad de 117,83, y por la parte baja, la década de 1960/69 con 857 nacimientos y una tasa de 94,33.

La baja tasa media de masculinidad que registra la población sileña en la centuria está en línea con la que se observa en los estudios sobre otras poblaciones. Por ejemplo, el estudio realizado por Stern (mencionado por Sánchez Compadre, 1989: 59) sobre la población negra norteamericana que registra valores de 102,6 e incluso inferiores a 100, o la de la población de Cabrera que registra una tasa media para el periodo de 1900/1969 del 101,48 (Quesada 2002: 81).

\section{III.- GEMERALIDAD}

De los 10793 partos registrados en Siles para el siglo XX, 97 han sido gemelares: 32 de dos niñas, 41 de dos niños, y 24 niño y niña ${ }^{16}$.

En la tabla 4 se puede observar que, en la población de Siles, las décadas del siglo XX que registran los índices de gemelaridad máximos han sido las dos primeras junto con la de 1940/49, y los mínimos en las dos últimas, con unos mínimos secundarios en las décadas de 1930/39 y 1920/29. Se aprecia que en la década de 1920/29 los nacimientos repuntan un 6,3\%, con relación a la anterior, mientras que los partos gemelares descienden un 47,3\%. Contrariamente, en la década de 1940/49 el número de nacimientos sileños desciende un $3 \%$ mientras los partos gemelares repuntan un 100\%. Fuster et al. (2009: 4) sugieren que en las épocas con una fecundidad alta una mayor natalidad no tiene por qué corresponder con una gemeralidad más elevada, explicando que esta relación inversa se da en la primera parte de la transición de la natalidad, y que el descenso de fecundidad se produce en las mujeres de mayor edad.

\footnotetext{
${ }^{16}$ La elevada frecuencia de nacimientos gemelares del mismo sexo con relación a los de distinto sexo provocó que se volviera a revisar las fotos de las actas de nacimientos con el fin de confirmar la fiabilidad de los datos tomados
} 
El brusco descenso de los partos gemelares sileños a partir de la década de 1970/79 se estima que ha sido causado principalmente por la emigración, que ha ocasionado la bajada del número de mujeres en edad de procrear y por tanto el número de concepciones. En estos 30 años de finales de siglo la tasa bruta de nupcialidad y la edad media al matrimonio se mantienen. No se tiene constancia de que alguno de los 2 partos gemelares registrados en la década de 1980/9 haya sido a consecuencia de un tratamiento de reproducción asistida o Fecundación In Vitro (FIV). En la década de 1990/99 no se registra un flujo emigratorio significativo, por lo que la ausencia de partos gemelares puede deberse al descenso de la nupcialidad (el 26,4\% con relación a su anterior década) y al aumento de la edad media de entrada al matrimonio (en los varones aumentó un $6 \%$ y en las mujeres un 7,7\%). Contrariamente a Siles, Fuster (2006: 23) registra para la población española una tasa creciente de gemelaridad en los últimos 20 años del siglo, que relaciona con el retraso en las edades de maternidad y la introducción a partir del año 1984 de las técnicas de reproducción asistida. Se deduce que la influencia del factor de la malnutrición en la frecuencia de partos gemelares sileños no ha sido significativa, dado que las décadas de la guerra y la posguerra, 1930/39 y 1940/49, registran relativamente un número alto de partos de este tipo.

Con respecto a la proporción secundaria de sexos de los nacimientos gemelares sileños se observa una tasa de masculinidad del 120,4, un 17,3\% más elevada que la de los partos simples. Alfonso-Sánchez y Peña (2003: 58) registran en la comarca de Lanciego una tasa del 119,2 para el periodo 1800/1990. Fellman y Ericsson (2009: 101) registran en Suecia, para el periodo de 1869/2004, una tasa de masculinidad en los partos gemelares del 103,2, un 2,5\% menos que la tasa de los partos simples. Igualmente, Jacobsen et al. (1999: 3122) encuentran en la población danesa de la década de los años 80 y principios de los 90 que en la proporción secundaria por sexos de los nacimientos gemelares disminuye la tasa de masculinidad. Ambos autores relacionan la menor tasa de masculinidad de los partos gemelares con una mayor mortalidad prenatal de los fetos varones.

Comparando los índices de gemeralidad sileños resultantes con los índices medios que registran las poblaciones humanas (Valls, 1972: 235) se observa que el índice de gemelos monozigóticos sileño no se mantiene constante y el 0,004539 supera el valor medio de 0,0035-0,0040. Con relación a los índices de gemeralidad dizigóticos apreciamos que no son constantes y que el 0,004447 sileño es muy inferior 
al 0,008 de la población caucasoide. Alfonso-Sánchez y Peña (2003: 55) ofrecen unos rangos de gemelaridad para las poblaciones rurales españolas. La frecuencia de gemelos dizigóticos obtenida para la población de Siles $(m=0,00444=4,44 \%$ ) se encuentra en la parte baja del rango proporcionado de $4,25-8,68 \%$. Mientras la frecuencia de gemelos monozigóticos sileña de $4,539 \%$ se sitúa muy por encima del rango ofrecido de $0,57-2,49 \%$.

En la tabla 5 se observa que la frecuencia monozigótica registrada en Siles es muy superior a la encontrada en las otras zonas rurales y algo superior a la hallada para España; solo el valor que registra la provincia de Jaén se acerca al sileño. Por el contrario, la frecuencia dizigótica sileña es de las más bajas, solamente las poblaciones de Ancares y Lanciego, entre las comparadas, registran un índice dizigótico menor. Con relación a los índices totales de gemeralidad se observa que la frecuencia encontrada en Siles se sitúa por el medio de la poblaciones comparadas, con cuatro que registran resultados inferiores (índices de menor a mayor: Ancares, Lanciego, Cabrera, Enciendo y Sanabria) y seis con resultados superiores (índices de menor a mayor: Babia, España, Alpujarra, Maragatería y Jaén). Los resultados obtenidos por Fuster et al. (2009: 3) para la población española (similares a los registrados por Valls) han mostrado escasa variación entre los años 1900 y 1980, manteniéndose dentro de un rango comprendido entre 7,3 y 10,1 por mil partos, con ligeras elevación de los índices a finales de los años 20 y en la década de los años 40. Los resultados registrados en la población sileña para estos 80 años presentan un rango comprendido entre $4,3 \%$ en la década 1970/79 y 12,6\% en la década 1910/19 y coinciden con los españoles en el repunte de la década 1940/49. Fuster et al. (2009: 6 y 2006: 19) indican que la variación de la tasas de gemeralidad entre las diferentes poblaciones puede estar influida por una evolución demográfica regional heterogénea, y que viene determinada principalmente por la frecuencia de gemelos dizigóticos dado que las frecuencias monozigóticas se mantienen constantes. Como se observa en la población de Siles, al igual que en las comarcas de los Ancares (Rodríguez, 1984) y de Lanciego ( Alfonso-Sánchez y Peña, 2003), estas últimas no se muestran constantes y varían de una década a otra debido a que se contabilizan un número bajo de partos gemelares.

Publicado en formato digital: Ramón Beteta Avio. TRANSICIÓN DE LA NATALIDAD DE SILES (Jaén) EN EL SIGLO XX: Tasa bruta, proporción secundaria por sexos, gemeralidad, ilegitimidad y estacionalidad. Revista Geográfica Digital. IGUNNE. Facultad de Humanidades. UNNE. Año 14. № 27. Enero - Junio 2017. ISSN 1668-5180 Resistencia, Chaco.

En: http://hum.unne.edu.ar/revistas/geoweb/default.htm 
Revista Geográfica Digital. IGUNNE. Facultad de Humanidades. UNNE. Año 14. № 27. Enero - Junio 2017. ISSN 1668-5180 Resistencia, Chaco

\section{IV.- ILEGITIMIDAD}

Los nacimientos de ilegítimos en la población sileña para el siglo XX han sido 636 (320 mujeres y 316 varones) de los 10793 nacimientos registrados, el 5,89\%. Se han incluido los registros en los que el cura anota que son hijos naturales pero añade el nombre de ambos padres y abuelos; también se incluyen a los legitimados por matrimonio posterior.

En la evolución de la natalidad ilegitima de Siles a lo largo del siglo XX (tabla 6) se registran continuas oscilaciones y dos etapas claramente diferenciadas:

La primera etapa comprendería desde 1900 a 1960 con una natalidad de ilegítimos relativamente elevada; en estas 6 décadas nace el 96,85\% del total de hijos fuera del matrimonio. Este periodo registra dos importantes subidas en las décadas de $1920 / 29$ y 1940/49, en las que los porcentajes repuntan respectivamente el $71,5 \%$ y el $51,6 \%$ sobre sus correspondientes décadas anteriores. También figuran dos significativos descensos en las décadas siguientes a las que se registraron los mayores repuntes, las de 1930/39 y 1950/59 en la que los porcentajes caen respectivamente el $42 \%$ y el $79,2 \%$. Se estima que las oscilaciones de las tasas de ilegítimos en esta primera etapa son producto de la inestabilidad social y económica. Se observa que las décadas que registran los mayores repuntes de ilegítimos coinciden en registrar en sus correspondientes quinquenios anteriores un repunte significativo de la mortalidad (en el de la "gripe española" el índice de mortalidad repuntó el 14,6\% sobre el lustro anterior, y en el de la Guerra Civil el 2.6\%). También, las tasas de ilegítimos se han visto afectadas por un aumento en el porcentaje de la población soltera y el retraso en la edad de entrada al matrimonio (las décadas de los años 40 y 50 son las que registran la edad media más elevada al matrimonio del siglo, unos 30 años para el varón y 26 para la mujer). Asimismo, el número de ilegítimos en esta primera etapa está relacionado con los bajos niveles de instrucción de la población (el $77,87 \%$ de los varones y el $91,65 \%$ de las mujeres eran analfabeto/as en el censo de 1900; el de 1940 todavía registra un 49\% de los varones y un 60,8\% de las mujeres). Además, se considera que puede existir una pequeña proporción de hijos ilegítimos causada por el comportamiento anómico de la mujer para "cazar" marido. No se observa una relación significativa entre el grado de religiosidad de la población y el número de ilegítimos, ya que los índices más elevados se dan en las décadas con mayor influencia religiosa. Se entiende que el nivel de nupcialidad afecta al grado de soltería que a su vez influye en las frecuencias de ilegítimos, siguiendo esta lógica, se 
observa que en la población de Siles el nivel de nupcialidad no influye en el número de ilegítimos, dado que los matrimonios repuntan en la década de 1920/29 el 27,6\%, con relación a su década anterior, coincidiendo con el mayor repunte de nacimientos ilegítimos, igualmente, en el segundo repunte significativo de la década 1940/49 el número de matrimonios también repunta el 2,3\%.

La segunda etapa estaría formada por las últimas cuatro décadas del siglo, en ella se registra solamente el $3,15 \%$ de los nacimientos ilegítimos de la centena. Este periodo registra un repunte en la década de 1980/89 y su posterior descenso en la década siguiente; como se puede observar en la tabla 6 , pasa de registrarse 2 ilegítimos en la década de 1970/79 a 7 en 1980/89 y descender en la década siguiente a 1. Las bajas tasas de ilegitimidad de la segunda etapa están relacionadas con el descenso de la población joven (los menores de 15 años pasaron de ser el 23,9\% de la población en el censo de 1981 al 14,4\% en el de 2001), el uso de anticonceptivos y la mayor posibilidad de abortar ${ }^{17}$.

En la segregación por sexos de la natalidad ilegítima sileña para el total de la centuria se observa que ambos registran similar número de nacimientos, con una mínima diferencia a favor de las mujeres. En la primera etapa, la década que registra mayor diferencia entre ambos sexos es la de 1920/29 en la que nacen 84 niñas ilegitimas (56\%) por 66 niños (44\%). En la segunda, las diferencias porcentuales entre ambos sexos son mayores que en la primera, no obstante, las desigualdades en el número de nacimientos ilegítimos son mucho menores, por ejemplo, en la década de $1970 / 79$ se registran dos nacimientos de varones que representan el $100 \%$ de ilegítimos de la década.

En el tabla 7 se pueden observar las frecuencias porcentuales de nacimientos ilegítimos de diversas poblaciones. Rodríguez (1984) en su estudio sobre la población de Ancares registra una tasa global de nacimientos ilegítimos similar a la sileña (la proporción sileña para el periodo de 1900/1970 es de 5,39). Entre ambas se aprecia una notable diferencia en la década de 1960/69, en la que la tasa de ilegitimidad de la población de Siles cae bruscamente mientras en Ancares se incrementa, para caer súbitamente en la década posterior al igual que la sileña solo que una década después. Este autor relaciona las tasas de ilegitimidad ancaresas con la ausencia de

\footnotetext{
17 Siguiendo los datos expuestos por Delgado (2000: 68) la tasa de aborto (por mil individuos) en Andalucía pasó de 0, 29 en 1987 a 4,20 en 1994 para el grupo de edad de entre 15 y 49 años.
} 
lugares de diversión en el invierno, la vida de pastoreo y los altos niveles de analfabetismo. Sánchez Compadre (1989) registra en Babia unas proporciones de ilegitimidad muy elevadas que relaciona con la permisividad social y religiosa. Alfonso y Peña (2003) hallan en Lanciego unas tasas de nacimientos fuera del matrimonio notablemente bajas y, a diferencia del resto de las poblaciones comparadas, registra un importante repunte en la década de la guerra civil. También observan cómo a partir del año 1940 dejan de registrarse nacimientos ilegítimos en esta población. Gil Alonso (1997: 44) observa mayor fecundidad urbana que rural en los grupos de edad de menores de 25 años que atribuye, entre otros factores, a una mayor incidencia de las concepciones prenupciales y que estas se convertían en polo de atracción en caso de embarazo prematrimonial de las mujeres rurales.

\section{IV.- ESTACIONALIDAD DE LOS NACIMIENTOS Y LAS CONCEPCIONES}

En la tabla 8 se exponen los resultados de la estacionalidad de los nacimientos sileños. El test Chi cuadrado registra variaciones estacionales significativas para el total del la centena $\left(x^{2}=151,730\right)$ y para cada una de las veintenas $\left(1900-1919, x^{2}=\right.$ 62,$799 ; 1920-1939, \quad X^{2}=108,768 ; 1940-1959, \quad X^{2}=28,305 ; 1960-1979, \quad x^{2}=$ 106,500; $1980-1999, X^{2}=50,091$; los valores mínimos para aceptar un patrón estacional significativo son $X^{2} 19,675 ; P=<0,05 ; 11$ g.l.).

En los nacimientos sileños por veintenas se observa que el patrón estacional ha ido variando de un periodo a otro. La primera veintena, 1900/19, registra unos coeficientes de estacionalidad máximos en agosto, y unos secundarios en marzo y septiembre; por tanto, las concepciones registran sus máximos en noviembre y diciembre. La segunda veintena, 1920/39, registra los coeficientes más elevados en febrero, marzo y mayo; y las concepciones registran los máximos en mayo, junio y agosto. En la tercera veintena, 1940/59, los máximos se registran en abril y mayo; por tanto, las concepciones presentan sus máximos en julio y agosto. A partir de la cuarta veintena, 1960/79, la estacionalidad de los nacimientos sileños es más homogénea debido al descenso de la natalidad; esta veintena presenta los coeficientes de estacionalidad más altos en los meses de julio y agosto y los de las concepciones en octubre y noviembre. El mayor número de concepciones en los meses otoñales de la primera y cuarta veintena está relacionado con la vuelta de los jornaleros agrícolas de la vendimia y la finalización de las demás faenas agrícolas que ocasionaban un periodo de poca actividad laboral (aún no se ha iniciado la recolección de la aceituna),

Publicado en formato digital: Ramón Beteta Avio. TRANSICIÓN DE LA NATALIDAD DE SILES (Jaén) EN EL SIGLO XX: Tasa bruta, proporción secundaria por sexos, gemeralidad, ilegitimidad y estacionalidad. Revista Geográfica Digital. IGUNNE. Facultad de Humanidades. UNNE. Año 14. № 27. Enero - Junio 2017. ISSN 1668-5180 Resistencia, Chaco.

En: http://hum.unne.edu.ar/revistas/geoweb/default.htm 
y con la tradicional "matanza" del cerdo que generaba ambientes festivos entre los convecinos. Los repuntes de las concepciones en los meses de primavera y verano de la segunda y tercera veintena están relacionados con el aumento de la temperatura y de las horas de luz de la primavera, y con las fiestas patronales, las vacaciones y el ocio en el verano. Asimismo, son previas a la emigración temporal de los jornaleros a la vendimia, por lo que se dan en una relativa calma laboral.

Sánchez Barricarte (2001: 140) menciona como en las poblaciones de Sangüesa y Yesa se buscaba que los nacimientos fuesen en las estaciones con menor carga de tareas agrícolas para así disponer de tiempo para la atención al parto y al cuidado del recién nacido, y que la mujer estuviera repuesta para ayudar en las estaciones con más trabajo. De forma similar, en estas cuatro veintenas del siglo un porcentaje de los nacimientos sileños en primavera y verano han sido elegidos de forma deliberada por la relativa calma de trabajo agrícola, al tiempo se buscaba que la mujer estuviera repuesta para trabajar en la temporada de aceituna y que el niño fuese algo mayor a la hora de afrontar la crudeza del invierno que se da en esta zona de montaña,

Estas cuatro primeras veintenas del siglo coinciden en registrar los coeficientes de estacionalidad mínimos en los meses finales del año, noviembre y diciembre. Por tanto, las concepciones registran los mínimos en los meses de febrero y marzo. Estos coeficientes mínimos están relacionados con la alta actividad agrícola, con el respeto a la cuaresma por ciertos sectores de la población y con la emigración temporal de los trabajadores de la madera fuera de la comarca, principalmente a los Pirineos.

La quinta veintena, 1980/1999, registra los coeficientes de estacionalidad más altos en los meses de octubre y junio; por tanto, los máximos en las concepciones se registran en enero y septiembre, dos meses con elevado trabajo agrícola (recolección de la aceituna y de la uva). Esto es indicativo de cómo en las décadas finales del siglo se produce una pérdida de influencia del sector primario en la economía de la población a consecuencia del crecimiento del sector terciario, hecho que ha ocasionado la diversificación de la estructura socio-profesional de la población, y por tanto, el descenso de influencia del factor de las labores agrícolas en las concepciones. En estas décadas el desarrollo socio-económico y la popularización de los métodos anticonceptivos hacen que los factores que más influyen en la estacionalidad de las concepciones sean el azar y la libre elección de los padres. 
La conocida relación existente entre la estacionalidad de los matrimonios y la de las concepciones no se puede observar con claridad porque no se dispone de información sobre el nacimiento de los primogénitos. No obstante, en la primera veintena se observa cierta relación dado que el mayor número de nacimientos en el mes de agosto puede estar conectado con el máximo de matrimonios en el mes de noviembre ${ }^{18}$.

Los coeficientes de estacionalidad hallados sobre la totalidad de los nacimientos sileños para el siglo XX muestran los máximos en el mes de agosto y los mínimos en los meses de noviembre y diciembre. La estacionalidad de la natalidad sileña presenta diferencias en los meses que registran los valores máximos con los observados en otras poblaciones como la Alpujarra (Luna, 1984), y Valdepeñas de Jaén (Quesada, 2000) con máximos en febrero y marzo; Babia (Sánchez-Compadre 1989) con máximos en marzo, abril, mayo y junio; los Ancares (Rodríguez, 1984), Sangüesa, Vera de Bidasoa y Yesa (Sánchez Barricarte, 2001) con máximos en los meses de marzo y abril. En cambio, los máximos veraniegos de Siles tienen similitud a los registrados en Casares de las Hurdes donde García-Moro (1986) los observa en septiembre y agosto, y a los registrados por Martínez Carrión (1984) en Yeste. Con relación a los mínimos, los registrados en Siles en los meses finales del año son similares a los observados por Luna (1984) en la Alpujarra, Sánchez Compadre (1989) en Babia, Quesada (2000) en Valdepeñas de Jaén o Martínez Carrión (1984) en Yeste. Difieren a los mínimos registrados por García-Moro (1986) en Casares de las Hurdes que los observa en el inicio de la primavera, o a los mínimos veraniegos registrados por Rodríguez (1984) en los Ancares y Sánchez Barricarte (2001) en Sangüesa, Vera de Bidasoa y Yesa. Al observar los nacimientos por semestres (tabla 8) se aprecia un mayor número de nacimientos sileños en la primera parte del año. Tanto Luna (1984: 72) en la Alpujarra como Rodríguez (1984: 97) en los Ancares también registran mayor número de nacimientos en el primer semestre. Finalmente, mencionar el estudio de Hernández et al. (2013) sobre la isla chilena de Pascua, en el que se presentan resultados de ausencia de estacionalidad debido a que el clima no registra cambios importantes (sin estaciones, lo que influye en la actividad tradicional

\footnotetext{
${ }^{18}$ La primera veintena del siglo, 1900-19, el mes de noviembre registra el $27,5 \%$ de los matrimonios, cuando en una distribución igualitaria entre los meses le correspondería el 8,33\%. Esta veintena registró 153 matrimonios en el mes de noviembre por 314 nacimientos en el mes de agosto.
} 
de obtención de alimentos) y la falta de influencia de las actividades religiosas y festivas.

La distribución mensual de los partos gemelares sileños registra los coeficientes estacionales máximos en primavera y mínimos en otoño. Rodríguez (1984: 107) registra en Ancares una estacionalidad de las frecuencias gemelares con máximos en invierno y mínimos en primavera y verano.

En la distribución mensual de los nacimientos sileños fuera del matrimonio los resultados obtenidos muestran los coeficientes estaciónales máximos en los meses de abril, junio, septiembre y agosto; y los mínimos en los meses finales del año. Las diferencias encontradas resultaron significativas ( $\mathrm{X} 2=35,847 ; \mathrm{gl} .11 ; \mathrm{P}=<0,001)$. Al relacionar la estacionalidad de los nacimientos ilegítimos con la estacionalidad de la natalidad general se observa que son coincidentes en los meses que registran los coeficientes estaciónales mínimos; en los máximos, coinciden agosto y junio y difieren en el mes de septiembre. Este repunte de nacimientos ilegítimos septiembre estaría relacionado con concepciones en las fiestas de Navidad y Año Nuevo. Rodríguez (1984: 104) en Ancares registra los máximos nacimientos ilegítimos en abril y enero y los mínimos, coincidentes con los registrados en la población sileña, en noviembre y diciembre. Las máximas las relaciona con las principales romerías que se celebran en el entorno de la población.

\section{CONCLUSIONES}

El número de nacimientos en Siles se mantiene por encima de los 1500 por década en la primera mitad del siglo. A partir del quinquenio 1950/54 inicia un descenso que lo lleva a registrar los mínimos en la última década del siglo. Se considera que el factor que más ha influido en la bajada de la natalidad sileña ha sido el flujo emigratorio de gran parte de sus habitantes. El elevado éxodo de personas, iniciado en la década de los años 40 , ha causado el despoblamiento y la disminución de los nacimientos y, en consecuencia, que el número de residentes sileños se redujese más de la mitad a finales de siglo y se incrementase su edad media, lo pone en cuestión la reproducción futura de la población sileña desde el punto de vista de su renovación y mantenimiento en el tiempo

En la población de Siles del siglo $X X$ se constata el mayor número de nacimientos varones. Se registra para la centuria una tasa de masculinidad del 102,65. 
El índice de gemeralidad sileño para la centuria (8,98\%) se sitúa por debajo de la media española (9,5\%). La tasa dizigótica de 4,447\% se sitúa en la parte baja del rango de variación para las poblaciones rurales españolas, mientras que la de monozigóticos de 4,539\%。 se encuentra muy por encima. A partir de 1970 los partos gemelares son excepcionales.

Las tasas sileñas de nacimientos de hijos fuera del matrimonio son bajas, lo que es indicativo del predominio de normas familiares tradicionales. Asimismo, se puede concluir que a partir de la década de 1960/69 los nacimientos ilegítimos no han influido significativamente en la natalidad general sileña.

Se observa que el patrón de estacionalidad de la natalidad sileña ha variado con el transcurrir del siglo XX, y que los factores más influyentes han sido los periodos de trabajo y ocio.

\section{BIBLIOGRAFÍA}

AGUINAGA ROUSTAN, J. (1989): "Descenso de la fecundidad y modernización en la sociedad española. Análisis comparativo de las encuestas de fecundidad 1977 y 1985". Boletín de la Asociación de Demografía Histórica (ADEH), vol. VII, nํ3, pp. 722

ALFONSO SÁNCHEZ, M. A. y PEÑA, J. A. (2003): "Tasas de gemeralidad e ilegitimidad en una comunidad rural del País Vasco (Lanciego: 1800-1990)" Antropo, 5, 55-62. Disponible en la WEB www.didac.ehu.es/antropo

ARANGO, J. (1980): "La teoría de la transición demográfica y la experiencia histórica". Revista Española de Investigaciones sociológicas (REIS) no 10, abril-junio, pp. 169198.

ARAQUE JIMENEZ, E. (1988): La Sierra de Segura: contribución al estudio de la crisis de la montaña andaluza. Tesis no publicada. Universidad de Granada.

ARROYO PÉREZ, A. et al. (1999): Un siglo de demografía en Andalucía: la población desde 1900. Instituto de Estadística de Andalucía. Sevilla.

BERTRANPETIT, J. (1978): "Evolución del tamaño de la población y natalidad en la isla de Formentera". En Actas del I Simposio de Antropología Biológica de España. Madrid. pp. 409-417.

BERTRANPETIT, J. y SALA, E. (1987): "Parámetros demográficos y proporción de sexos". Homenaje al Dr. José María Basabe. Eusko-lkaskuntza. Sociedad de Estudios Vascos. Donostia - San Sebastián, pp. 171-178. 
BRIAN, E. y JAISSON, M. (2005): "El sexismo en las estadísticas de los nacimientos. Reflexiones sobre una investigación de Halbwachs en los años 1930". Revista de Metodología de Ciencias Sociales, № 9, enero-junio, pp.11-38

CAMAROTA S. A. (2007): "Nación ilegitima”. Center for Inmigration Studies. Disponible en www.cis.org

CLARKIN, P. (2011): The sex ratio at birth following periods of conflict. En la web: www.kevishere.com

DELGADO, M. (2000): La fecundidad joven y adolescente en España. Editorial Universidad de Granada. Granada.

DEL REY, A., CEBRIÁN, M. y ORTEGA J. A. (2009): "Despoblamiento y envejecimiento en Castilla y León durante el siglo $\mathrm{XX}$ : análisis a través de la emigración femenina y la perdida de nacimientos". Revista de Estudios sobre Despoblamiento y Desarrollo Rural. Ager, № 8. Zaragoza. pp. 111-179

DEVOLDER, D., NICOLAU, R. y PANAREDA, E. (2006): "La fecundidad de las generaciones españolas nacidas en la primera mitad del siglo XX. Un estudio a escala provincial" Revista de Demografía Historica, XXIV, I, segunda temporada, pp. 57-89

DÍEZ MEDRANO, J. (1984): "Reflexiones teóricas sobre la evolución de la ilegitimidad en Europa (1945- 1984)". En Revista Española de Investigaciones Sociológicas (REIS), 27/84 pp. 79-106. Disponible en la Web: www.reis.cis.es>REIS>PDF>REIS_27_06_pdf

EGEA, C. (1999): La población de loa municipios de Jaén: evolución en el siglo XX y situación actual. Diputación Prov. de Jaén. Instituto de Estudios Giennenses.

FELLMAN, J. y ERIKSSON, A. W. (2006): Weinberg's Differential Rule Reconsidered. Human Biology, June 2006, vol. 78, nº 3, pp. 253-275.

FELLMAN, J. y ERIKSSON, A. W. (2009): "Secondary sex ratio in multiple births". Twin Research and Human Genetics, vol. 13, no 1, pp. 101-108.

FUSTER, V, DE BLAS, C., COLANTONIO, S. y ALONSO, V. (2006): "Evolución y variabilidad de las tasas de gemeralidad en la población española". Revista Española de Antropología Física, 26: 19-24

FUSTER, V., ROMÁN-BUSTO, J., ZULUAGA, P. y COLANTONIO, S. E. (2009) "Análisis histórico de la gemeralidad en España: Diferencias regionales". Antropo, 20, 1-9. www.didac.ehu.es/antropo. 
FOSCHIATTI, A. M. (2010): "La natalidad y la Fecundidad". Resúmenes. Revista geográfica digital. IGUNNE. Facultad de Humanidades. UNNE. Año 7, № 13. Enerojunio. Resistencia, Chaco. En: http://hum.unne.edu.ar/revista/geoweb/default.htm GARCIA-MORO, C. (1986): Entre brezos y colmenas. (La población de Casares de las Hurdes en los siglos XVI al $X X)$. Editorial Regional de Extremadura .

GIL ALONSO, F. (1997): "Las diferencias territoriales en el descenso de la fecundidad en España". Boletín de la Asociación de Demografía Histórica, XV, II, pp. 13-54.

HENRY, L. (1976): Demografía. Ed. Labor. Barcelona.

HENRY, L. (1983): Manual de demografía histórica. Ed. Crítica. Barcelona.

HERNÁNDEZ, M., GARCÍA MORO, C. y ESPARZA, M. (2013): "Ausencia de estacionalidad de los nacimientos en la isla de Pascua". Revista Española de Antropología Física, vol. 34: 35-41

HAWLEY, A. H. (1962): Ecología humana. Ed. Tecnos. Madrid. 1962.

INFANTE AMATE, J. (2011): "Los temporeros del olivar. Una aproximación al estudio de las migraciones estacionales en el sur de España (siglos XVIII-XX)". Revista de Demografía Histórica, XXIX, II, segunda época, pp. 87-117

INSTITUTO NACIONAL DE ESTADÍSTICA (1956): Reseña Estadística de la Provincia de Jaén. Presidencia del Gobierno. Madrid.

JACOBSEN, R., MOLLER, H. Y MOURITSEN, A. (1999): "Natural variation in the human sex ratio". Human Reproduction, vol. 14, no12, pp. 3120-3125.

LIVI-BACCI, M. (1993): Introducción a la demografía. Ariel. Barcelona.

LOSADA ÁLVAREZ, A. (1999): "Cuba (1898-1958). Descenso de la fecundidad y cambio socioeconómico". Boletín de la Asociación de Demografía Histórica, 1999 XVII, 1, pp. 41-78.

LUNA, F. (1984): Demografía de la Alpujarra. Estructura y biodinámica. Universidad de Granada. Granada

MARTINEZ CARRIÓN, J. M. (1984): Estacionalidad y cambio demográfico. La transformación del "ciclo vital anual" en tierras albacetenses durante los silgo XIX y XX.Web:Bibliteca2.uclm.es/biblioteca/CECLM/ARTREVISTA/ALBASIT/Alb13Martinez. pdf . pp. 87-136.

NADAL, J. (1984): La población española (Siglos XVI a XX). Ed. Ariel. Barcelona. QUESADA, A. y GARCÍA, C.J. (2000): "Estacionalidad de los nacimientos en una población andaluza". En Investigaciones en biodiversidad humana, Universidad de Santiago de Compostela. pp. 130-136 
QUESADA, A. (2002): Biodemografía de una población andaluza. Valdepeñas de Jaén (1841-1992). Tesis no publicada. Universidad de Granada. Granada.

RODRÍGUEZ OTERO, H. (1984): Bioantropología de la comarca de los ancares leoneses. Diputación provincial de León. León

SÁNCHEZ BARRICARTE, J. J. (2001): "El comportamiento reproductivo en tres municipios navarros (siglos XVIII al XX)". Revista de Demografía Histórica, XIX, II, segunda época, pp. 115-143

SÁNCHEZ COMPADRE, E. (1989): BABIA. Biodemografía y estructura familiar. Secretariado de publicaciones. Universidad de León.

SANZ, A. y GONZÁLEZ, F. R. (2001): "Las mujeres y el control de la fecundidad. Propuesta metológica para su identificación durante la transición demográfica". Revista de Demografía Histórica, XIX, II, segunda época, pp.57-78

VALLS, A. (1972). "Sobre la repartición de la gemeralidad en España". Revista Española de Antropología Americana, 7: 2. Facultad de filosofía y letras. Universidad de Madrid. pp:235-244. 
Evolución anual del número de nacimientos. Siles, 1900 - 1999

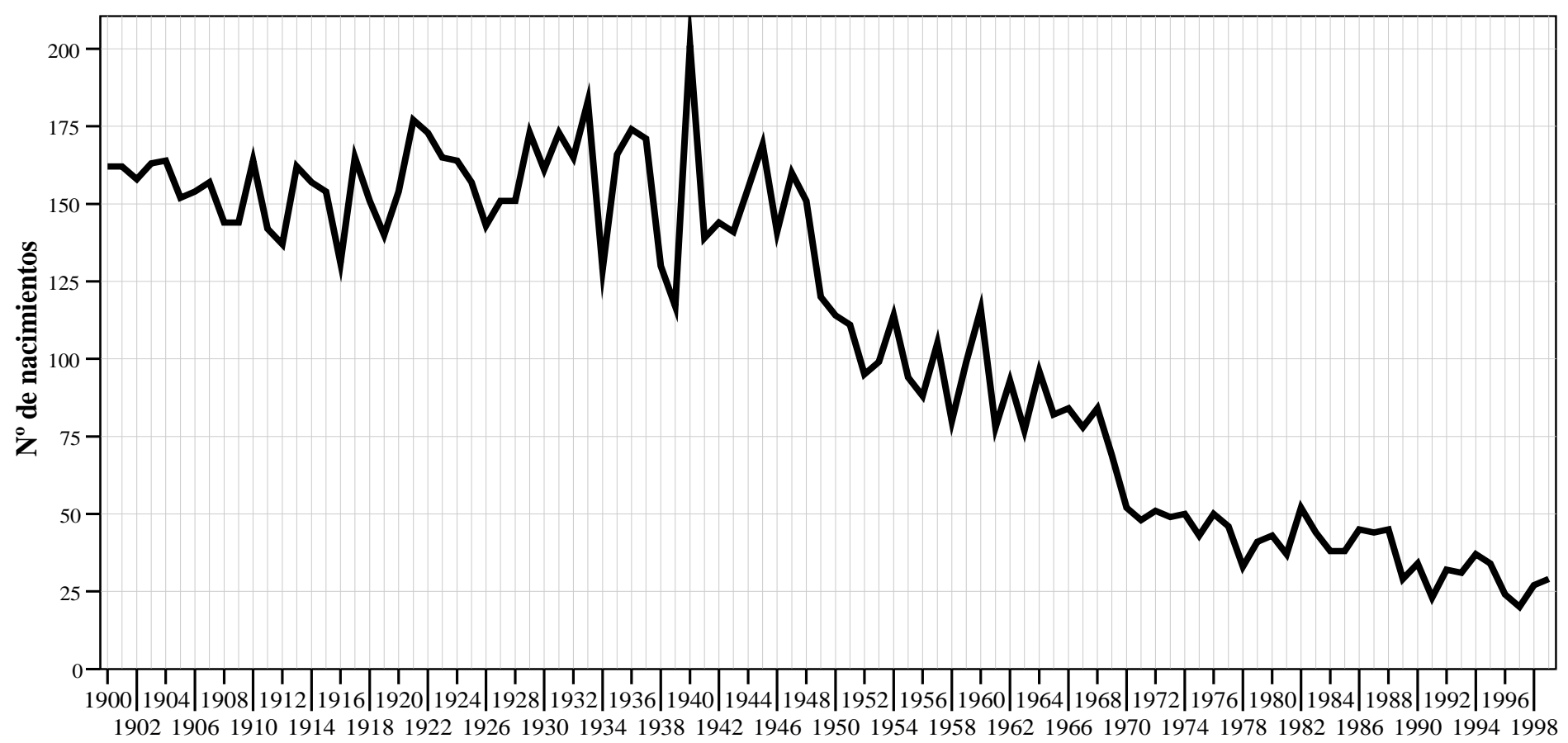

Años

Figura 1 
Revista Geográfica Digital. IGUNNE. Facultad de Humanidades. UNNE. Año 14. № 27.

Enero - Junio 2017. ISSN 1668-5180 Resistencia, Chaco

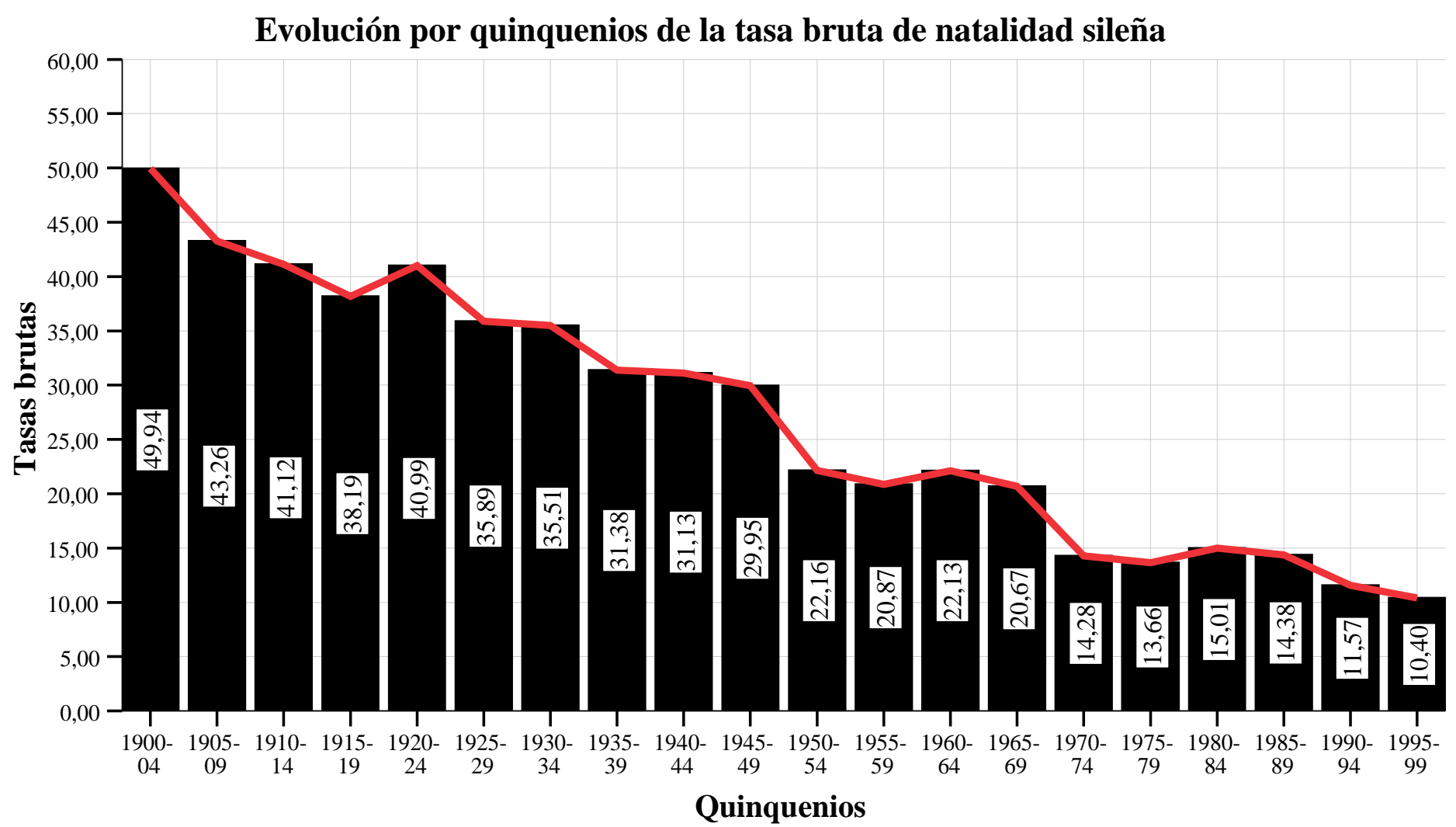

Figura 2

Tabla 1.- Evolución de las tasas brutas de natalidad de diversas poblaciones

\begin{tabular}{|c|c|c|c|c|c|c|c|c|c|c|}
\hline & Siles & $\begin{array}{l}\text { Sile } \\
s \\
(1)\end{array}$ & $\begin{array}{l}\text { Sierra } \\
\text { Segur } \\
\text { a }\end{array}$ & $\begin{array}{l}\text { Jaén } \\
\text { Prov. }\end{array}$ & Andalucía & $\begin{array}{l}\text { Españ } \\
\text { a }\end{array}$ & $\begin{array}{l}\text { Jaén } \\
\text { capital }\end{array}$ & $\begin{array}{l}\text { Ancare } \\
\mathrm{s}\end{array}$ & Babia & Alpujarra \\
\hline \multirow{2}{*}{$\begin{array}{l}1900- \\
1909\end{array}$} & $\begin{array}{l}49,9 \\
4\end{array}$ & \multirow{2}{*}{41,1} & \multirow{2}{*}{45} & $\begin{array}{l}40,3 \\
0\end{array}$ & 37,26 & 34,86 & 39,86 & \multirow{2}{*}{32,5} & \multirow{2}{*}{24,24} & 54,4 \\
\hline & $\begin{array}{l}43,2 \\
6\end{array}$ & & & $\begin{array}{l}39,2 \\
7\end{array}$ & 36,06 & 33,66 & 38,54 & & & 55,21 \\
\hline \multirow{2}{*}{$\begin{array}{l}1910- \\
1919\end{array}$} & $\begin{array}{l}41,1 \\
2\end{array}$ & \multirow{2}{*}{38} & \multirow{2}{*}{37,1} & $\begin{array}{l}37,1 \\
7\end{array}$ & 34,41 & 31,16 & 36,20 & \multirow{2}{*}{25,19} & \multirow{2}{*}{21,36} & 49,98 \\
\hline & $\begin{array}{l}38,1 \\
9\end{array}$ & & & $\begin{array}{l}34,6 \\
3\end{array}$ & 31,90 & 29,08 & 33,80 & & & 45,17 \\
\hline $\begin{array}{l}1920- \\
1929\end{array}$ & $\begin{array}{l}40,9 \\
9\end{array}$ & 37,9 & 37,5 & $\begin{array}{l}35,0 \\
5\end{array}$ & 33,10 & 29,96 & 36,51 & 31,76 & 22,9 & 40,04 \\
\hline
\end{tabular}


Revista Geográfica Digital. IGUNNE. Facultad de Humanidades. UNNE. Año 14. № 27. Enero - Junio 2017. ISSN 1668-5180 Resistencia, Chaco

\begin{tabular}{|c|c|c|c|c|c|c|c|c|c|c|}
\hline & $\begin{array}{l}35,8 \\
9\end{array}$ & & & $\begin{array}{l}36,2 \\
8\end{array}$ & 32,28 & 28,66 & 35,13 & & & 34,59 \\
\hline \multirow{2}{*}{$\begin{array}{l}1930- \\
1939\end{array}$} & $\begin{array}{l}35,5 \\
1\end{array}$ & \multirow{2}{*}{33} & \multirow{2}{*}{33,4} & $\begin{array}{l}34,2 \\
1\end{array}$ & 31,71 & 27,48 & 33,32 & \multirow{2}{*}{30,24} & \multirow{2}{*}{20,97} & 35,88 \\
\hline & $\begin{array}{l}31,3 \\
8\end{array}$ & & & $\begin{array}{l}29,8 \\
1\end{array}$ & 25,80 & 21,86 & 30,33 & & & 30,86 \\
\hline \multirow{2}{*}{$\begin{array}{l}1940- \\
1949\end{array}$} & $\begin{array}{l}31,1 \\
3\end{array}$ & & & $\begin{array}{l}27,6 \\
0\end{array}$ & 25,66 & 21,78 & 25,39 & \multirow{2}{*}{19,7} & \multirow{2}{*}{19,94} & 30,23 \\
\hline & $\begin{array}{l}29,9 \\
5\end{array}$ & & & $\begin{array}{l}27,1 \\
8\end{array}$ & 25,70 & 21,82 & 26,93 & & & 29,05 \\
\hline \multirow{2}{*}{$\begin{array}{l}1950- \\
1959\end{array}$} & $\begin{array}{l}22,1 \\
6\end{array}$ & & & $\begin{array}{l}24,7 \\
0\end{array}$ & 23,10 & 20,16 & & \multirow{2}{*}{15,37} & \multirow{2}{*}{20,62} & 26,15 \\
\hline & $\begin{array}{l}20,8 \\
7\end{array}$ & & & $\begin{array}{l}25,5 \\
6\end{array}$ & 24,43 & 21,20 & & & & 24,00 \\
\hline \multirow{2}{*}{$\begin{array}{l}1960- \\
1969\end{array}$} & $\begin{array}{l}22,1 \\
3\end{array}$ & & & $\begin{array}{l}26,3 \\
4\end{array}$ & 24,72 & 21,38 & & \multirow{2}{*}{13,68} & \multirow{2}{*}{14,86} & 23,68 \\
\hline & $\begin{array}{l}20,6 \\
7\end{array}$ & & & $\begin{array}{l}21,9 \\
0\end{array}$ & 23,27 & 20,46 & & & & 20,52 \\
\hline \multirow{2}{*}{$\begin{array}{l}1970- \\
1979\end{array}$} & $\begin{array}{l}14,2 \\
8\end{array}$ & & & $\begin{array}{l}18,0 \\
1\end{array}$ & 21,16 & 19,38 & & \multirow{2}{*}{6,1} & \multirow{2}{*}{10,72} & 15,42 \\
\hline & $\begin{array}{l}13,6 \\
6\end{array}$ & & & $\begin{array}{l}16,1 \\
9\end{array}$ & 19,65 & 17,74 & & & & 11,69 \\
\hline \multirow{2}{*}{$\begin{array}{l}1980- \\
1989\end{array}$} & $\begin{array}{l}15,0 \\
1\end{array}$ & & & $\begin{array}{l}15,3 \\
9\end{array}$ & 16,73 & 13,56 & & & & \\
\hline & $\begin{array}{l}14,3 \\
8\end{array}$ & & & $\begin{array}{l}13,6 \\
0\end{array}$ & 13,89 & 11,12 & & & & \\
\hline \multirow{2}{*}{$\begin{array}{l}1990- \\
1999\end{array}$} & $\begin{array}{l}11,5 \\
7\end{array}$ & & & $\begin{array}{l}12,8 \\
7\end{array}$ & 12,42 & 9,98 & & & & \\
\hline & $\begin{array}{l}10,4 \\
0\end{array}$ & & & $\begin{array}{l}11,0 \\
6\end{array}$ & 10,86 & 9,28 & & & & \\
\hline
\end{tabular}

Fuentes: Siles (1) y Sierra de Segura, Araque (1988: 148); España, Nadal (1984: 140-141) hasta el año 1980, años posteriores INEbase; Andalucía y provincia Jaén, Web del IECA; ciudad de Jaén, INE (1956: 92); Ancares, Rodríguez (1984: 93); Babia, Sánchez Compadre (1989: 55); y Alpujarra, Luna (1984: 65) 


\section{Evolución de la natalidad de España, Andalucía, Jaén prov. y Siles promediada para periodos de 5 años}

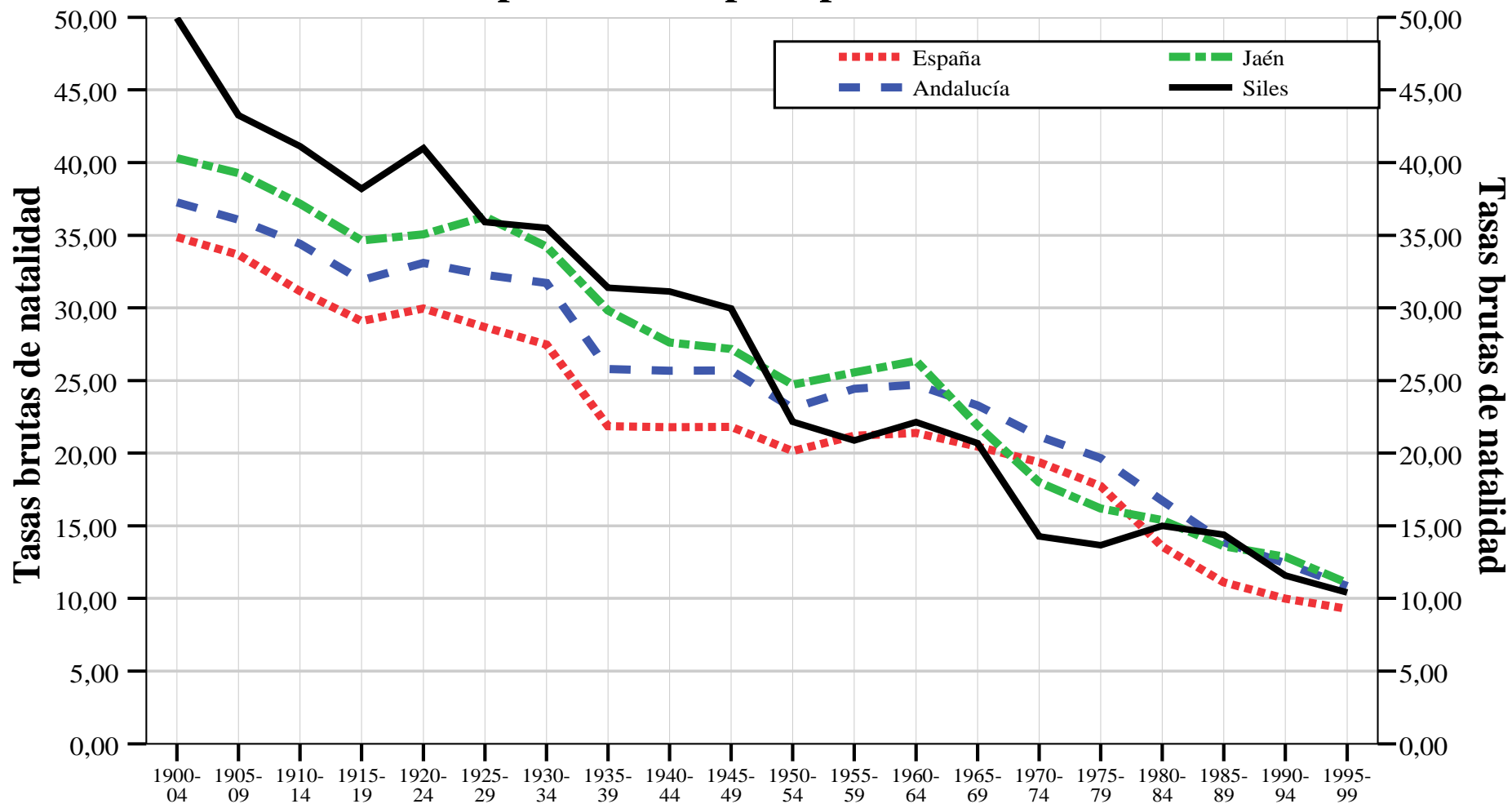

\section{Quinquenios}

Figura 3

\begin{tabular}{|l|l|l|l|}
\hline $\begin{array}{l}\text { Tabla 2.- } \\
\text { décadas }\end{array}$ \\
\hline DÉCADAS & MUJERES & VARONES & TOTAL \\
\hline $1900-09$ & 777 & 784 & 1561 \\
\hline $1910-19$ & 690 & 813 & 1503 \\
\hline $1920-29$ & 820 & 788 & 1608 \\
\hline $1930-39$ & 791 & 778 & 1569 \\
\hline $1940-49$ & 776 & 745 & 1521 \\
\hline $1950-59$ & 472 & 527 & 999 \\
\hline $1960-69$ & 441 & 416 & 857 \\
\hline & & &
\end{tabular}


Revista Geográfica Digital. IGUNNE. Facultad de Humanidades. UNNE. Año 14. № 27. Enero - Junio 2017. ISSN 1668-5180 Resistencia, Chaco

\begin{tabular}{|l|l|l|l|}
\hline $1970-79$ & 232 & 231 & 463 \\
\hline $1980-89$ & 202 & 213 & 415 \\
\hline $1990-99$ & 141 & 150 & 291 \\
\hline TOTAL & 5342 & 5445 & 10787 \\
\hline
\end{tabular}

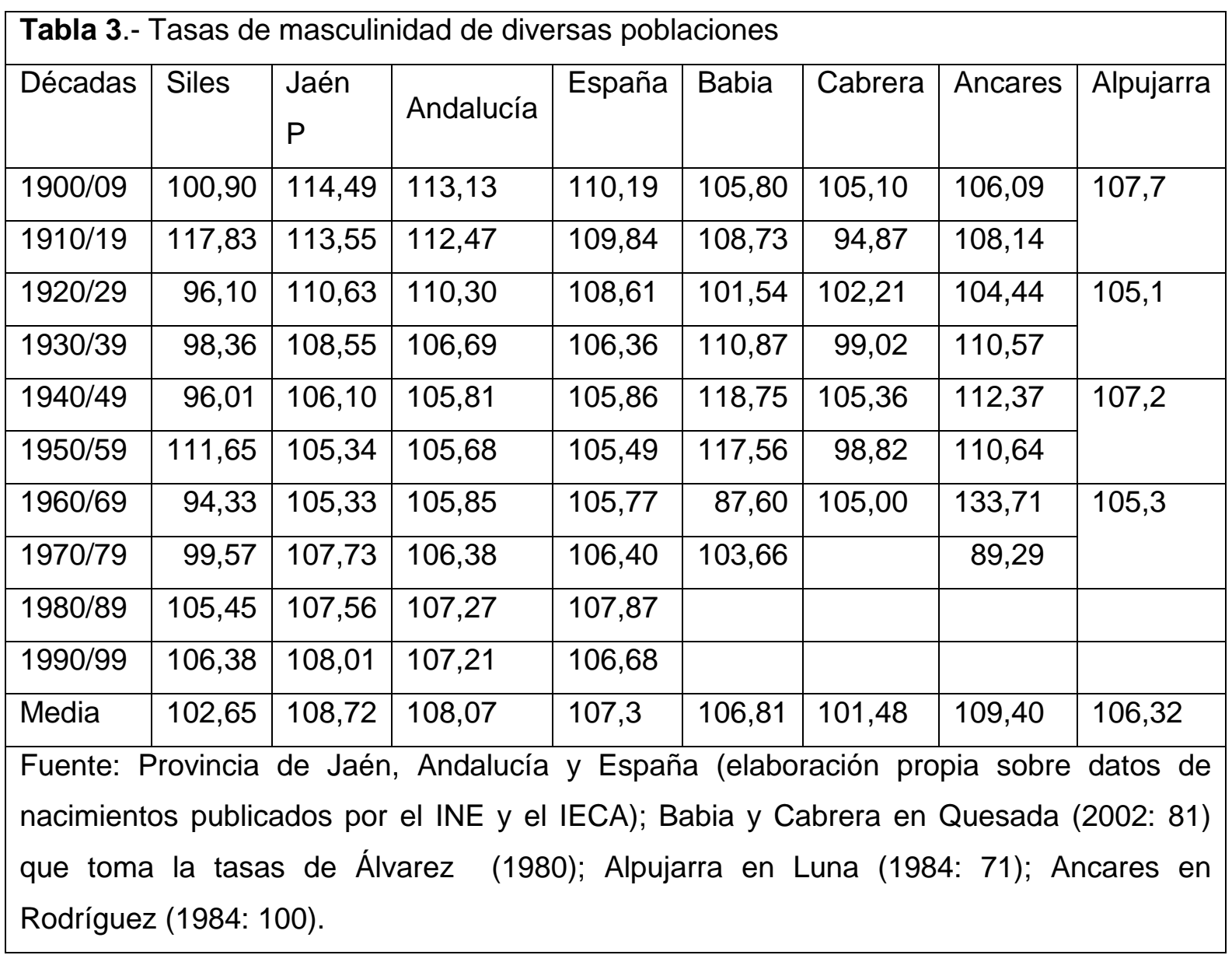

Tabla 4.- Frecuencias gemelares por décadas en Siles 
Revista Geográfica Digital. IGUNNE. Facultad de Humanidades. UNNE. Año 14. № 27.

Enero - Junio 2017. ISSN 1668-5180 Resistencia, Chaco

\begin{tabular}{|c|c|c|c|c|c|c|c|c|c|}
\hline \multirow[b]{2}{*}{ Décadas } & \multicolumn{3}{|c|}{$\begin{array}{ll}\text { Partos } & \text { gemelares } \\
\text { Monozigóticos } & \end{array}$} & \multicolumn{2}{|c|}{$\begin{array}{l}\text { Partos gemelares } \\
\text { Dizigóticos }\end{array}$} & \multicolumn{2}{|c|}{ Partos gemelares } & \multirow{2}{*}{$\begin{array}{l}\text { Total } \\
\text { sileños }\end{array}$} & \multirow[t]{2}{*}{ Nacimientos } \\
\hline & $\begin{array}{l}\text { Niña } \\
-a\end{array}$ & $\begin{array}{l}\text { Niño } \\
-0\end{array}$ & índice & $\begin{array}{l}\text { Niña- } \\
\text { o }\end{array}$ & índice & $\mathrm{N}$ & índice & & \\
\hline 1900-09 & 5 & 7 & 0.003839 & 6 & 0.007678 & 18 & 0.011516 & 1563 & \\
\hline 1910-19 & 6 & 11 & 0.009960 & 2 & 0.002656 & 19 & 0.012616 & 1506 & \\
\hline $1920-29$ & 5 & 4 & 0.004975 & 1 & 0.001244 & 10 & 0.006219 & 1608 & \\
\hline 1930-39 & 4 & 1 & 0.000637 & 4 & 0.005099 & 9 & 0.005736 & 1569 & \\
\hline 1940-49 & 7 & 7 & 0.006575 & 4 & 0.005260 & 18 & 0.011834 & 1521 & \\
\hline 1950-59 & 2 & 6 & 0.006006 & 2 & 0.004004 & 10 & 0.010010 & 999 & \\
\hline $1960-69$ & 2 & 3 & 0.001167 & 4 & 0.009335 & 9 & 0.010502 & 857 & \\
\hline 1970-79 & - & 1 & 0.002155 & 1 & 0.002155 & 2 & 0.004310 & 464 & \\
\hline 1980-89 & 1 & 1 & 0.004819 & - & - & 2 & 0.004819 & 415 & \\
\hline $1990-99$ & - & - & 0.003839 & - & - & - & 0.000000 & 291 & \\
\hline $1900-1999$ & 32 & 41 & 0.004539 & 24 & 0.004447 & 97 & 0.008986 & 10793 & \\
\hline
\end{tabular}

\begin{tabular}{|c|c|c|c|c|c|}
\hline Población & Periodo & Monozigóticos & Dizigóticos & $\begin{array}{l}\text { Total } \\
\text { Gemelar }\end{array}$ & Fuentes \\
\hline Alpujarra & 1900- 1978 & 0.001579 & 0.008391 & 0.009970 & Luna (1984: 84) \\
\hline Babia & 1850- 1979 & 0.000570 & 0.008680 & 0.009250 & \multirow{3}{*}{$\begin{array}{l}\text { En Sánchez Compadre } \\
(1989: 60)\end{array}$} \\
\hline Cabrera & - & 0.002220 & 0.006150 & 0.008370 & \\
\hline Sanabria & - & 0.002480 & 0.006480 & 0.008960 & \\
\hline Ancares & 1870- 1979 & 0.001952 & 0.004259 & 0.006211 & \multirow{3}{*}{$\begin{array}{l}\text { En Rodríguez Otero } \\
(1984: 108)\end{array}$} \\
\hline Encinedo & 1890- 1969 & 0.002228 & 0.006143 & 0.008371 & \\
\hline Maragatería & $1900-1970$ & 0.002492 & 0.007937 & 0.010429 & \\
\hline Lanciego & 1800- 1999 & 0.003976 & 0.004372 & 0.0083500 & $\begin{array}{l}\text { Alfonso-Sánchez y Peña } \\
\text { (2003: 58) }\end{array}$ \\
\hline España & 1951- 1967 & 0.003500 & 0.005990 & 0.009490 & \multirow{2}{*}{ Valls (1972: 238 y 243) } \\
\hline Prov. Jaén & 1951- 1967 & 0.004270 & 0.007750 & 0.012020 & \\
\hline Siles & $1900-1999$ & 0.004539 & 0.004447 & 0.008986 & \\
\hline
\end{tabular}

Tabla 6.- Frecuencias absolutas y porcentajes de nacimientos ilegítimos en Siles

\begin{tabular}{|l|l|l|l|l|l|}
\hline & Mujeres & Varones & Total & Nacimientos & Porcentajes \\
\hline $1900-09$ & 43 & 38 & 81 & 1563 & 5.18 \\
\hline
\end{tabular}

Publicado en formato digital: Ramón Beteta Avio. TRANSICIÓN DE LA NATALIDAD DE SILES (Jaén) EN EL SIGLO XX: Tasa bruta, proporción secundaria por sexos, gemeralidad, ilegitimidad y estacionalidad. Revista Geográfica Digital. IGUNNE. Facultad de Humanidades. UNNE. Año 14. № 27. Enero - Junio 2017. ISSN 1668-5180 Resistencia, Chaco.

En: http://hum.unne.edu.ar/revistas/geoweb/default.htm 
Revista Geográfica Digital. IGUNNE. Facultad de Humanidades. UNNE. Año 14. № 27. Enero - Junio 2017. ISSN 1668-5180 Resistencia, Chaco

\begin{tabular}{|l|l|l|l|l|l|}
\hline $1910-19$ & 43 & 39 & 82 & 1506 & 5.44 \\
\hline $1920-29$ & 84 & 66 & 150 & 1608 & 9.33 \\
\hline $1930-39$ & 48 & 52 & 100 & 1569 & 6.37 \\
\hline $1940-49$ & 69 & 78 & 147 & 1521 & 9.66 \\
\hline $1950-59$ & 23 & 33 & 56 & 999 & 5.61 \\
\hline $1960-69$ & 7 & 3 & 10 & 857 & 1.17 \\
\hline $1970-79$ & 0 & 2 & 2 & 464 & 0.43 \\
\hline $1980-89$ & 2 & 5 & 7 & 415 & 1.69 \\
\hline $1990-99$ & 1 & 0 & 1 & 291 & 0.34 \\
\hline $1900-99$ & 320 & 316 & 636 & 10793 & 5.89 \\
\hline
\end{tabular}

Tabla 7.- Frecuencias porcentuales de nacimientos ilegítimos en algunas poblaciones

\begin{tabular}{|c|c|c|c|c|c|c|c|}
\hline & Siles & Ancares & Babia & $\begin{array}{l}\text { Casares } \\
\text { de las } \\
\text { Hurdes }\end{array}$ & Pereda & Luneras & Lanciego \\
\hline $1900-09$ & 5.18 & 7.40 & 16.61 & \multirow{5}{*}{2.8} & 2.83 & 8.51 & 1.33 \\
\hline $1910-19$ & 5.44 & 4.22 & 14.09 & & 2.80 & 7.48 & 0.95 \\
\hline $1920-29$ & 9.33 & 4.47 & 13.12 & & 0.92 & 11.11 & 0.43 \\
\hline 1930-39 & 6.37 & 3.39 & 13.56 & & - & 5.45 & 1.04 \\
\hline $1940-49$ & 9.66 & 6.76 & 10.11 & & 6.06 & 14.29 & 0.00 \\
\hline $1950-59$ & 5.61 & 6.38 & 6.24 & \multirow{3}{*}{2.9} & 6.45 & 14.00 & 0.00 \\
\hline 1960-69 & 1.17 & 6.73 & 4.60 & & 2.33 & 14.29 & 0.00 \\
\hline $1970-79$ & 0.43 & 1.89 & 3.59 & & 9.09 & 0.00 & 0.00 \\
\hline 1980-89 & 1.69 & - & - & - & - & - & 0.00 \\
\hline 1990-99 & 0.34 & - & - & - & - & - & - \\
\hline Medias & 4.52 & 5.15 & 10.24 & 2.85 & 3.81 & 9.39 & 0.41 \\
\hline
\end{tabular}

Fuentes: Ancares, Pereda y Luneras, Rodríguez (1984: 103); Babia, Sánchez Compadre (1989:

55); Casares de las Hurdes, García-Moro (1986: 76); Lanciego, Alfonso y Peña (2003: 59).

Tabla 8.- Número de nacimientos y coeficientes de estacionalidad sileños por veintenas

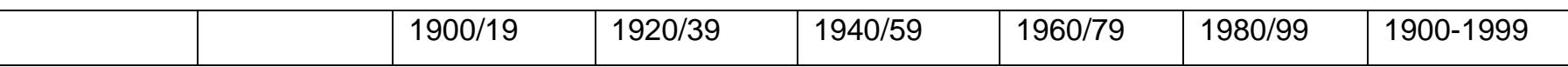

Publicado en formato digital: Ramón Beteta Avio. TRANSICIÓN DE LA NATALIDAD DE SILES (Jaén) EN EL SIGLO XX: Tasa bruta, proporción secundaria por sexos, gemeralidad, ilegitimidad y estacionalidad. Revista Geográfica Digital. IGUNNE. Facultad de Humanidades. UNNE. Año 14. № 27. Enero - Junio 2017. ISSN 1668-5180 Resistencia, Chaco.

En: http://hum.unne.edu.ar/revistas/geoweb/default.htm 
Revista Geográfica Digital. IGUNNE. Facultad de Humanidades. UNNE. Año 14. № 27.

Enero - Junio 2017. ISSN 1668-5180 Resistencia, Chaco

\begin{tabular}{|c|c|c|c|c|c|c|c|c|c|c|c|c|c|}
\hline $\begin{array}{l}\text { Mes } \\
\text { concepción }\end{array}$ & $\begin{array}{l}\text { Mes } \\
\text { nacimiento }\end{array}$ & $\mathrm{N}$ & Cei & $\mathrm{N}$ & Cei & $\mathrm{N}$ & Cei & $\mathrm{N}$ & Cei & $\mathrm{N}$ & Cei & $\mathrm{N}$ & Cei \\
\hline Abril & Enero & 237 & 0,93 & 256 & 0,97 & 227 & 1,08 & 108 & 0,98 & 65 & 1,10 & 893 & 0,99 \\
\hline Mayo & Febrero & 243 & 0,95 & 322 & 1,22 & 212 & 1,01 & 120 & 1,09 & 55 & 0,93 & 952 & 1,06 \\
\hline Junio & Marzo & 296 & 1,16 & 303 & 1,14 & 227 & 1,08 & 97 & 0,88 & 52 & 0,87 & 973 & 1,08 \\
\hline Julio & Abril & 265 & 1,04 & 290 & 1,10 & 232 & 1,10 & 112 & 1,01 & 57 & 0,97 & 955 & 1,06 \\
\hline Agosto & Mayo & 265 & 1,03 & 301 & 1,14 & 239 & 1,14 & 117 & 1,06 & 51 & 0,87 & 972 & 1,08 \\
\hline Septiem & Junio & 270 & 1,06 & 307 & 1,16 & 220 & 1,05 & 116 & 1,05 & 69 & 1,17 & 981 & 1,09 \\
\hline Octubre & Julio & 256 & 1,00 & 289 & 1,09 & 216 & 1,03 & 121 & 1,10 & 60 & 1,02 & 942 & 1,05 \\
\hline Noviemb & Agosto & 314 & 1,23 & 296 & 1,12 & 219 & 1,04 & 122 & $\overline{1,11}$ & 66 & 1,12 & 1017 & 1,13 \\
\hline Diciemb & Septiemb & 297 & 1,16 & 250 & 0,94 & 205 & 0,98 & 114 & 1,04 & 48 & 0,82 & 914 & 1,02 \\
\hline Enero & Octubre & 238 & 0,93 & 212 & 0,80 & 182 & 0,87 & 111 & 1,01 & 71 & 1,19 & 813 & 0,90 \\
\hline Febrero & Noviembr & 190 & 0,74 & 174 & 0,66 & 172 & 0,82 & 89 & 0,81 & 59 & 1,00 & 684 & 0,76 \\
\hline Marzo & Diciembr & 194 & 0,76 & 178 & 0,67 & 170 & 0,81 & 94 & 0,85 & 55 & 0,93 & 691 & 0,77 \\
\hline \multicolumn{2}{|l|}{ Semestre $1^{\circ}$} & 1576 & 6,17 & 1779 & 6,72 & 1357 & 6,46 & 670 & 6,08 & 349 & 5,92 & 5726 & 6,37 \\
\hline \multicolumn{2}{|l|}{ Semestre $2^{\circ}$} & 1489 & 5,83 & 1399 & 5,28 & 1164 & 5,54 & 651 & 5,92 & 359 & 6,08 & 5061 & 5,63 \\
\hline \multicolumn{2}{|l|}{ Totales } & \multicolumn{2}{|l|}{3065} & \multicolumn{2}{|l|}{3178} & \multicolumn{2}{|l|}{2521} & \multicolumn{2}{|l|}{1321} & \multicolumn{2}{|l|}{708} & \multicolumn{2}{|c|}{10793} \\
\hline
\end{tabular}

